\title{
Perivascular macrophages mediate the neurovascular and cognitive dysfunction associated with hypertension
}

\author{
Giuseppe Faraco, ${ }^{1}$ Yukio Sugiyama, ${ }^{1}$ Diane Lane, ${ }^{1}$ Lidia Garcia-Bonilla, ${ }^{1}$ Haejoo Chang, ${ }^{1}$ Monica M. Santisteban, ${ }^{1}$ \\ Gianfranco Racchumi, ${ }^{1}$ Michelle Murphy, ${ }^{1}$ Nico Van Rooijen, ${ }^{2}$ Joseph Anrather, ${ }^{1}$ and Costantino ladecola ${ }^{1}$ \\ 'Feil Family Brain and Mind Research Institute, Weill Cornell Medicine, New York, New York, USA. ²Department of Molecular Cell Biology, VU University Medical Center, Amsterdam, Netherlands.
}

\begin{abstract}
Hypertension is a leading risk factor for dementia, but the mechanisms underlying its damaging effects on the brain are poorly understood. Due to a lack of energy reserves, the brain relies on continuous delivery of blood flow to its active regions in accordance with their dynamic metabolic needs. Hypertension disrupts these vital regulatory mechanisms, leading to the neuronal dysfunction and damage underlying cognitive impairment. Elucidating the cellular bases of these impairments is essential for developing new therapies. Perivascular macrophages (PVMs) represent a distinct population of resident brain macrophages that serves key homeostatic roles but also has the potential to generate large amounts of reactive oxygen species (ROS). Here, we report that PVMs are critical in driving the alterations in neurovascular regulation and attendant cognitive impairment in mouse models of hypertension. This effect was mediated by an increase in blood-brain barrier permeability that allowed angiotensin II to enter the perivascular space and activate angiotensin type 1 receptors in PVMs, leading to production of ROS through the superoxide-producing enzyme NOX2. These findings unveil a pathogenic role of PVMs in the neurovascular and cognitive dysfunction associated with hypertension and identify these cells as a putative therapeutic target for diseases associated with cerebrovascular oxidative stress.
\end{abstract}

\section{Introduction}

Hypertension afflicts up to one-third of the world population and is a leading risk factor for morbidity and mortality worldwide (1). The brain is a major target organ of the damaging effects of hypertension (2). Well recognized as the most important risk factor for stroke and vascular cognitive impairment (3), hypertension has also been linked to Alzheimer disease, the leading cause of dementia in the elderly (4). Therefore, hypertension is implicated in major brain pathologies and remains a highly prevalent and potentially treatable cause of brain dysfunction and damage. Although treatment of elevated blood pressure (BP) has greatly reduced stroke mortality (5), its impact on cognitive dysfunction has been less clear (2), highlighting our limited understanding of the effects of hypertension on the brain.

The health of the cerebrovascular system is vital for the brain's functional and structural integrity. The brain has no energy reserves and requires a continuous supply of blood well matched to its dynamic and regionally diverse metabolic needs (6). Neurons, glia, and vascular cells, key components of the so-called neurovascular unit (NVU), work in concert to assure that the brain is always adequately perfused (6). Thus, brain activation increases cerebral blood flow $(\mathrm{CBF})$ to support the increased energy demands and

\section{Related Commentary: p. 4393}

Conflict of interest: The authors have declared that no conflict of interest exists. Submitted: February 5, 2016; Accepted: September 30, 2016

Reference information: / Clin Invest. 2016;126(12):4674-4689. doi:10.1172/JCI86950. remove potentially harmful by-products of cerebral metabolism, a process known as neurovascular coupling (7). At the same time, endothelial cells, the site of the blood-brain barrier (BBB), regulate the trafficking of molecules and cells between blood and brain (8), and coordinate microvascular flow by releasing vasoactive agents (9). Hypertension leads to profound cerebrovascular alterations (2). In addition to structural changes (hypertrophy, remodeling, stiffening, lipohyalinosis, etc.) (2), hypertension induces alterations in cerebrovascular regulation that promote vascular insufficiency (2). Thus, in humans as in animal models, hypertension disrupts all the major factors regulating the cerebral circulation, including neurovascular coupling and endothelial vasomotor function $(10,11)$. As a result, the brain becomes more susceptible to neuronal dysfunction and damage, which underlies vascular cognitive impairment (12).

The factors responsible for these functional alterations of the NVU are poorly understood, and their exploration is essential to develop preventative or therapeutic approaches to mitigate the impact of hypertension on brain health. Angiotensin II (ANGII) plays an important role in human hypertension and has been used extensively to explore the pathobiology of the disease (13). Administration of low doses of ANGII for 2 weeks, which results in a slow-developing rise in BP ("slow pressor" hypertension) (14), induces profound alterations in neurovascular coupling and endothelium-dependent vasodilation $(10,15)$. The cerebrovascular dysfunction is mediated by activation of ANGII type 1 receptors (AT1Rs) and vascular oxidative stress produced by a NOX2-containing NADPH oxidase $(10,15)$. The downstream mechanisms 

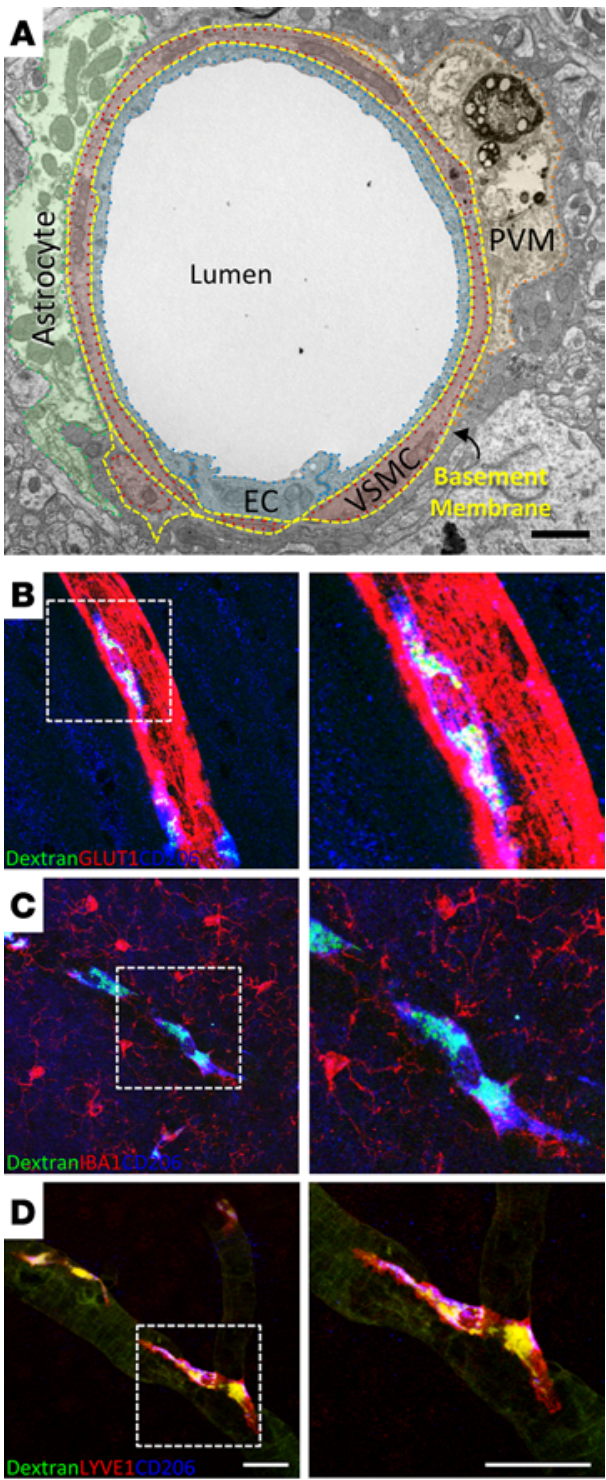

by which ANGII-induced oxidative stress alters cerebrovascular function involve nitrosative stress and NO depletion $(16,17)$. However, the vascular cell type(s) that produces reactive oxygen species (ROS) and initiates the dysfunction remains to be elucidated. Furthermore, it is unclear whether the neurovascular dysfunction is required for the development of cognitive deficits.

Perivascular macrophages (PVMs) and meningeal and choroid plexus macrophages represent the bulk of resident brain macrophages (18), and are distinct from macrophages infiltrating the wall of large vessels in inflammatory conditions, such as atherosclerosis (19). Residing in the intracerebral perivascular space, delimited by the glia limitans and the vascular basement membrane (Virchow-Robin space), PVMs are closely apposed to the outer vessel wall and originate from hematopoietic precursors (18, $20,21)$. As the vessels penetrate deeper into the substance of the brain, the glial and vascular basement membranes fuse together and the perivascular space disappears (22). As macrophages, PVMs express AT1Rs and have the potential to produce large amounts of ROS through NOX2 $(23,24)$.
Figure 1. PVM identification. (A) Electron micrograph of a neocortical arteriole (diameter $10 \mu \mathrm{m}$ ). A PVM containing lysosomes (electron dense) is seen at the top right of the vessel (scale bar: $1 \mu \mathrm{m}$ ). PVMs can be identified as perivascular cells (A-D), positive for CD206 (B-D), able to phagocytose i.c.V.-injected FITC-dextran (B-D), weakly positive for the microglia marker IBA1 (C), and expressing the endothelial lymphatic vessel marker LYVE1 (D). Scale bars: $25 \mu \mathrm{m}$

In this study we investigated the contribution of PVMs to the neurovascular and cognitive dysfunction induced by hypertension. We found that depletion of PVMs in models of chronic hypertension suppresses vascular oxidative stress and ameliorates the attendant impairment in neurovascular coupling and endothelium-dependent responses. Studies in bone marrow (BM) chimeras provided evidence that the dysfunction is mediated by ANGII acting on PVM AT1Rs resulting in NOX2-dependent ROS production. Importantly, concomitant to the neurovascular improvement, PVM depletion also rescued cognitive dysfunction. The findings unveil a previously unrecognized role of PVMs in the neurovascular and cognitive dysfunction induced by hypertension, and identify PVMs as a novel pathogenic component of the NVU of critical importance for brain health.

\section{Results}

Characterization of PVMs. First, we used well-established criteria to identify brain PVMs (18). Brain PVMs were identified as myeloid cells closely associated with the abluminal side of cerebral blood vessels and (a) expressing the mannose receptor CD206 (Figure 1, B-D, and ref. 18); (b) expressing lymphatic vessel endothelial hyaluronan receptor-1 (LYVE1) (ref. 25 and Figure 1D); (c) expressing low levels of IBA1, compared with microglial cells (Figure 1C); and (d) endowed of phagocytic activity assessed by injection of dextran into the lateral ventricles (Figure 1, B-D, and ref. 26). PVMs were found most abundantly in association with pial and parenchymal vessels larger than $20 \mu \mathrm{m}$ in diameter, particularly pial arterioles, a key site for flow regulation (7), the length of which was covered $70 \%-80 \%$ by PVMs (Supplemental Figure 1, A-E; supplemental material available online with this article; doi:10.1172/ JCI86950DS1).

Slow pressor ANGII disrupts the BBB, leading to ANGII entry into the perivascular space. To investigate the role of PVMs in the cerebrovascular dysfunction induced by ANGII hypertension, we first sought to determine whether circulating ANGII crosses the $\mathrm{BBB}$ and reaches the perivascular space. Infusion of low concentrations of ANGII (600 $\mathrm{ng} / \mathrm{kg} / \mathrm{min}$ ) induced a delayed increase in BP that was observed at day 7 and leveled off by day 14 (Figure 2A). At this time, ANGII concentration was increased in plasma (Figure 2B), but not in brain (Supplemental Figure 2, A-C). To determine whether plasma ANGII (MW $~ 1$ $\mathrm{kDa}$ ) is able to cross the BBB and gain access to the perivascular space, we examined the permeability of the BBB to sodium fluorescein (MW $0.3 \mathrm{kDa}$ ) and FITC-dextran (MW $3 \mathrm{kDa}$ ). We found that chronic ANGII infusion increased the permeability of the BBB to both markers at 14 days, whereas acute administration of ANGII (250 ng/ $\mathrm{kg} / \mathrm{min}$ ) produced a comparable elevation in mean BP, but failed to do so (Figure 2C and Supplemental Figure 2E). The increased permeability to FITC-dex- 

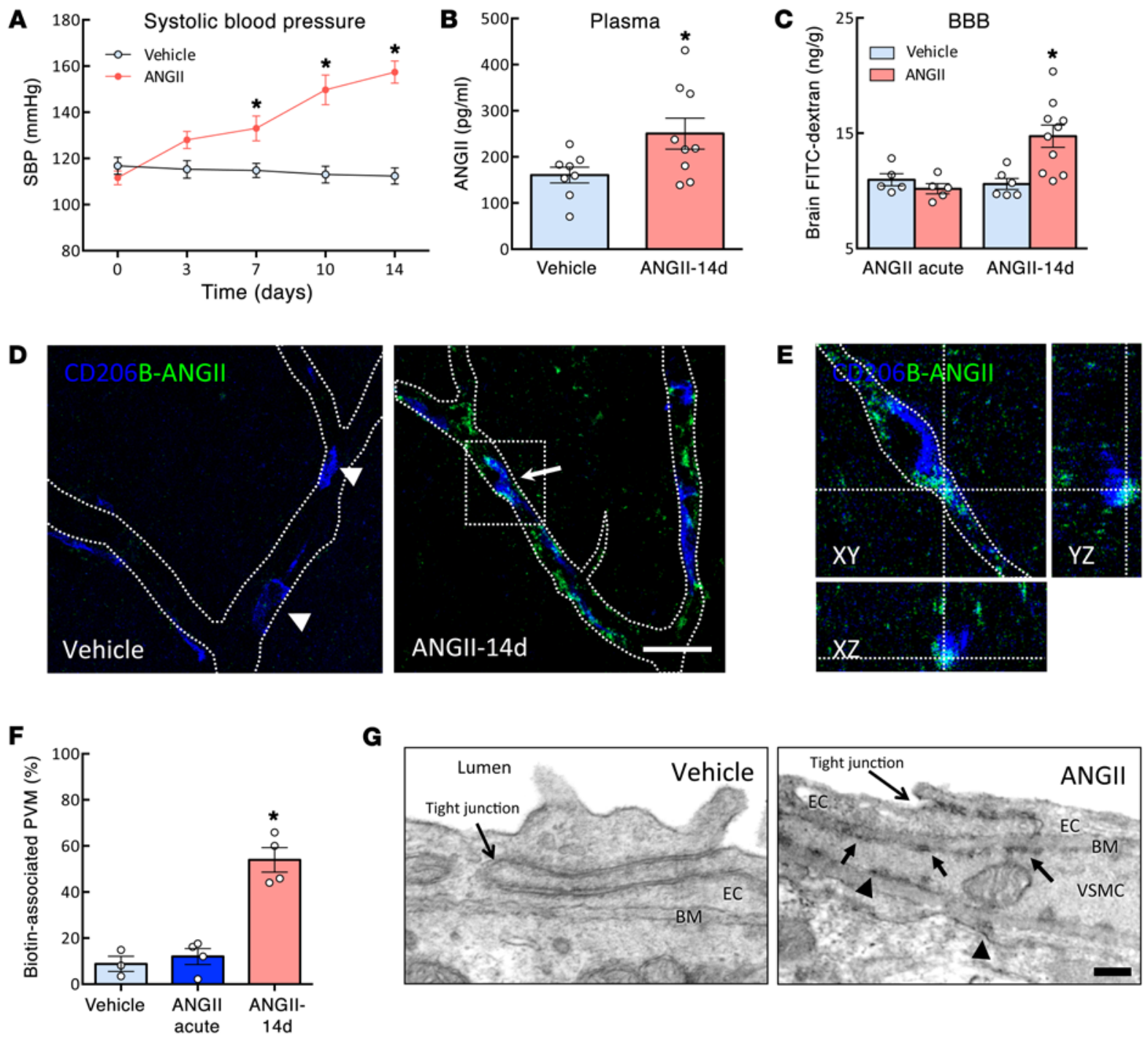

Figure 2. Slow pressor ANGII disrupts the BBB, leading to ANGII entry into the perivascular space and PVMs. (A) Slow pressor ANGIl gradually increases systolic blood pressure measured by tail-cuff plethysmography. ${ }^{*} P<0.05$ vs. vehicle (Veh); $n=12-17$ (2-way ANOVA and Bonferroni's test). (B) ANGIl plasma levels are increased after 2 weeks of ANGll administration. ${ }^{*} P<0.05$ vs. Veh; $n=8-9$ (Student's $t$ test). (C) Slow pressor hypertension but not acute i.v. ANGII administration increases BBB permeability to FITC-dextran (MW $3 \mathrm{kDa}$ ). ${ }^{*} P<0.05 \mathrm{vs}$. Veh; $n=5-7$ (Student's $t$ test). (D) Confocal microscopy showing immunofluorescence labeling of biotinylated ANGII around cerebral blood vessels and in association with PVMs in mice treated with ANGII for 14 days but not in saline-treated mice (scale bar: $25 \mu \mathrm{m}$ ). (E) Orthogonal views (XY, XZ, YZ) illustrating colocalization of biotin-ANGII (green) and CD206 (blue). (F) Biotin-ANGII is associated with PVMs in mice treated with ANGII for 14 days but not in saline-treated mice or in mice acutely infused with ANGII. ${ }^{*} P<0.05$ vs. Veh and acute ANGII; $n=3-4$ per group; $86 \pm 9$ PVMs per animal (1-way ANOVA and Tukey's test). (G) Electron micrographs of cortical arterioles showing immunoperoxidase labeling of biotinylated ANGII tracking along tight junctions (arrows) and reaching perivascular space (arrowheads) in mice treated with ANGII for 14 days (right) but not in saline-treated mice (left). Scale bar: $100 \mathrm{~nm}$. PVM, perivascular macrophage; EC, endothelial cell; VSMC, vascular smooth muscle cells; BM, basement membrane.

tran was observed prior to 14 days, but the effect did not reach statistical significance (Supplemental Figure 2F). In contrast to FITC-dextran and sodium fluorescein, the BBB permeability to Evans blue, which binds to serum albumin (MW $\sim 69 \mathrm{kDa}$ ), was not altered by chronic ANGII administration (Supplemental Figure 2E), indicating a previously unappreciated size restriction in the BBB dysfunction. The effect of ANGII on the BBB could not be attributed solely to the elevation in BP, since 2 weeks of phenylephrine infusion increased $\mathrm{BP}$ comparably to ANGII, but did not alter BBB permeability to FITC-dextran (Supplemental Figure 2, G and H).
To provide more direct evidence that blood-borne ANGII reaches the perivascular space in proximity to PVMs, we administered biotinylated ANGII i.v. on day 14 of the ANGII infusion, and used confocal and electron microscopy to detect the labeled peptide. As shown in Figure 2, D-F, nearly $60 \%$ of the PVMs were closely associated with biotinylated ANGII in mice treated with ANGII for 14 days (Figure 2, D-F). In contrast, little or no ANGII labeling was observed in mice implanted with pumps delivering saline or in which ANGII was administered acutely (Figure 2, D and F). Furthermore, by using electron microscopy, we demonstrated that ANGII-biotin, tracked along tight junctions, crossed 
A

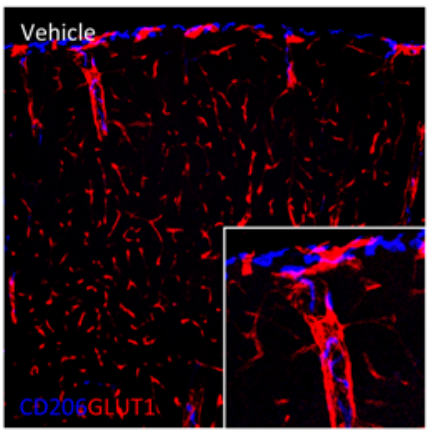

C

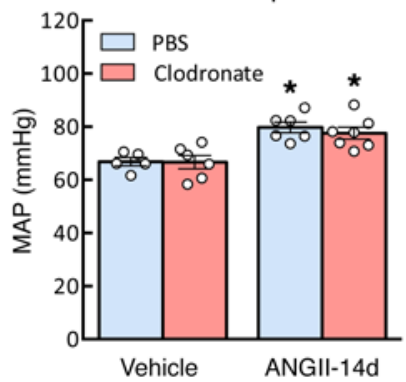

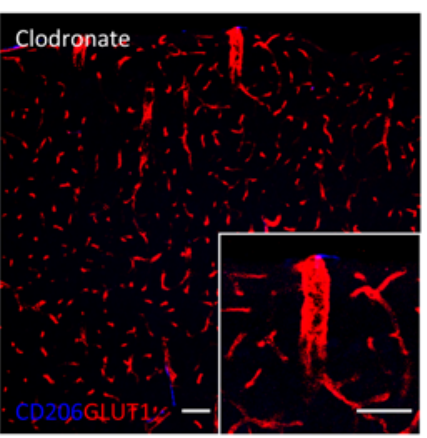

D

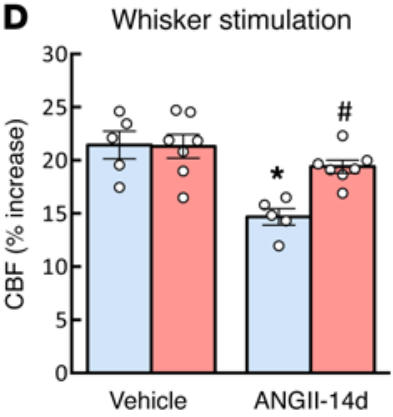

B

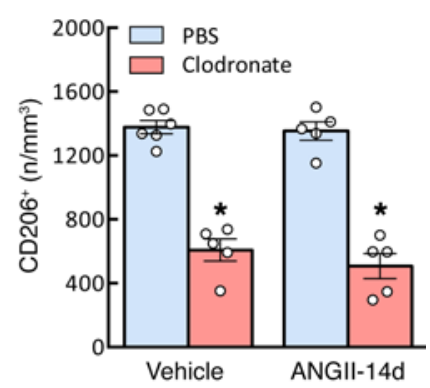

Acetylcholine

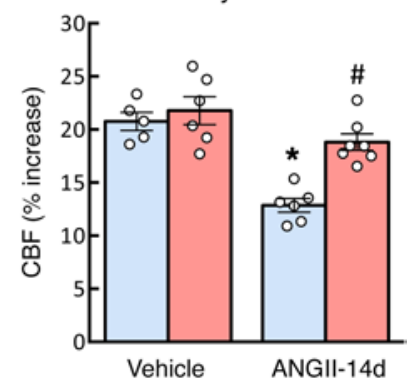

Figure 3. PVM depletion by CLOD restores neurovascular function in ANGIl slow pressor hypertension. (A and B) Intracerebroventricular CLOD administration induces depletion of cells expressing the PVM marker CD206 in the somatosensory cortex assessed by histology. The vasculature is visualized by double label with the endothelial marker GLUT-1. The pial surface is at the top of the figure, and penetrating vessels can be seen diving into the neocortex. Scale bars: $50 \mu \mathrm{m} .{ }^{*} P<0.05$ vs. PBS-Veh and PBS-ANGIl; $n=5-10$ per group (2-way ANOVA and Bonferroni's test). (C) CLOD administration does not affect the increases in mean arterial pressure (MAP) induced by ANGII. ${ }^{*} P<0.05$ vs. PBS-Veh and CLOD-Veh; $n=5-8$ per group (2-way ANOVA and Bonferroni's test). (D) CLOD does not alter the increase in CBF induced by whisker stimulation or cortical administration of ACh, but counteracts the attenuation of both responses induced by ANGII. ${ }^{*} P<0.05$ vs. PBS-Veh and CLOD-Veh; ${ }^{*} P<0.05$ vs. PBS-ANGII; $n=5-7$ per group ( 2 -way ANOVA and Bonferroni's test).

the endothelium and reached the perivascular space (Figure 2G). Thus, in the "slow pressor" model circulating ANGII is able to cross the BBB and reach PVMs in the perivascular space.

PVM depletion by clodronate restores neurovascular function in ANGII slow pressor hypertension. Since ANGII reaches the perivascular space, we asked whether PVMs contribute to the neurovascular dysfunction induced by ANGII hypertension. To this end, we depleted PVMs using liposome-encapsulated clodronate (CLOD) (27). CLOD is phagocytosed by macrophages, leading to their demise by apoptosis. Thus, administration of CLOD into the cerebral ventricles (i.c.v.) is widely used to deplete PVMs in brain $(28,29)$. In mice treated with vehicle (liposome-encapsulated PBS), PVMs, as described in Figure 1, were observed wrapping around cerebral blood vessels (Figure 3A). CLOD treatment selectively reduced PVMs $\left(\mathrm{CD} 206^{+} \mathrm{CD} 45^{\mathrm{hi}} \mathrm{CD} 11 \mathrm{~b}^{+}\right)$, but not brain macrophages (CD206 ${ }^{-} \mathrm{CD} 45^{\text {hi }} \mathrm{CD} 11 \mathrm{~b}^{+}$) (Figure 3, A and B, and Supplemental Figure 3, A and B). CLOD did not deplete microglial cells (Supplemental Figure 3C) or blood leukocytes (Supplemental Figure 3D).

Having established the effectiveness of CLOD, we then examined whether PVM depletion counteracts the cerebrovascular dysfunction induced by ANGII. CLOD or vehicle was administered i.c.v., and 7 days later mice were implanted with osmotic minipumps delivering ANGII or saline. Cerebrovascular function was assessed 2 weeks after minipump implantation. As before (10), slow pressor ANGII attenuated the CBF increase produced in the somatosensory cortex by mechanical stimulation of the whiskers (neurovascular coupling) and endothelium-dependent $\mathrm{CBF}$ responses to neocortical application of acetylcholine (ACh) (Figure 3D). Responses to adenosine were not affected (Supplemental Table 1), ruling out that the neurovascular dysfunction was due to failure of the vasomotor apparatus. CLOD depleted PVMs by $60 \%-65 \%$ in mice receiving vehicle or ANGII (Figure 3B), but did not alter the increase in BP (Figure 3C and Supplemental Figure 3E). Similarly, in vehicle-treated mice CLOD did not affect the CBF increases evoked by whisker stimulation, ACh, or adenosine (Figure 3D and Supplemental Table 1). However, in ANGII-treated mice CLOD counteracted the attenuation of the vascular responses (Figure 3D), implicating PVMs in the mechanisms of the dysfunction.

Acute i.v. administration of high concentrations of ANGII alters neurovascular function (11). Since ANGII needs to cross the BBB to reach the PVM, we hypothesized that the dysfunction induced by acute i.v. ANGII, which does not alter the BBB (Figure 2C), would be insensitive to PVM depletion. On the other hand, the neurovascular dysfunction caused by application of ANGII to the subarachnoid space underlying the cranial window (30), which bypasses the BBB and has direct access to the perivascular space, would be rescued by CLOD. Consistent with this prediction, CLOD counteracted the CBF dysfunction produced by subarachnoid application of ANGII, but not that induced by acute i.v. infusion of the peptide (Figure 4, A and B), attesting 
A

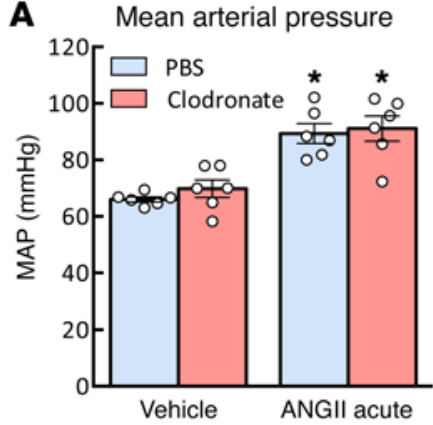

B

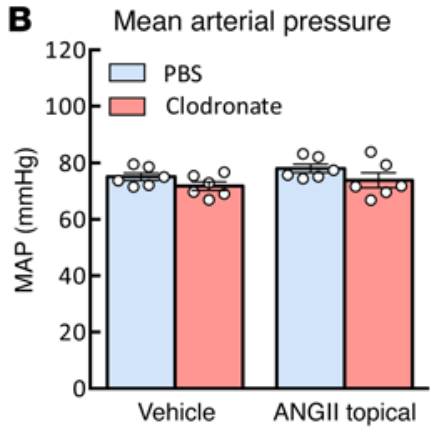

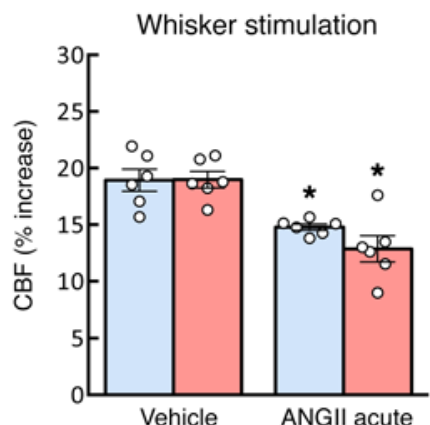

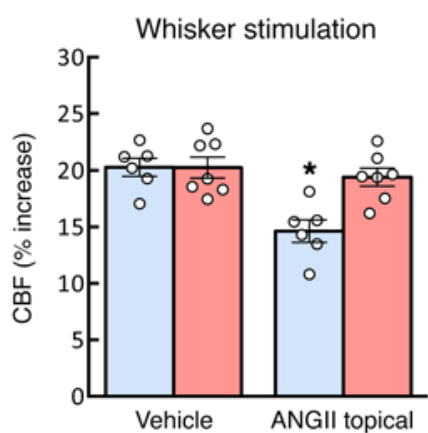

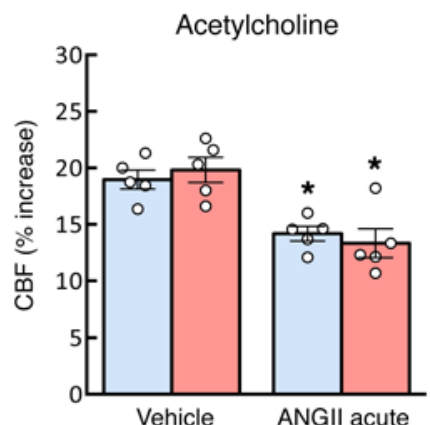

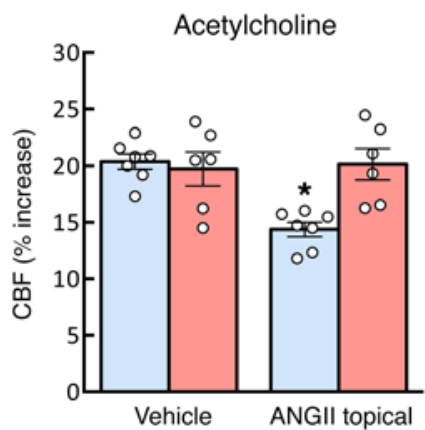

Figure 4. PVM depletion counteracts the neurovascular dysfunction induced by topical neocortical application but not by acute i.v. ANGII administration. (A) CLOD has no effect on the neurovascular dysfunction induced by acute i.v. administration of ANGII. ${ }^{*} P<0.05$ vs. PBS-Veh and CLOD-Veh; $n=6$ per group (2-way repeated-measures ANOVA and Bonferroni's test). (B) CLOD-mediated PVM depletion rescues the neurovascular dysfunction induced by neocortical application of ANGII to the subarachnoid space (ANGII topical). ${ }^{*} P<0.05$ vs. PBS-Veh and CLOD-Veh; $n=6-7$ per group (2-way repeated-measures ANOVA and Bonferroni's test).

to the specificity of the effect of PVM depletion. These observations indicate that PVMs are able to induce neurovascular dysfunction when exposed to ANGII.

PVM AT1Rs participate in the harmful cerebrovascular effects of ANGII. AT1Rs are involved in the cerebrovascular dysfunction induced by ANGII (10). Macrophages are well known to express AT1R and produce ROS in response to $\operatorname{ANGII}(23,24)$. Therefore, we sought to determine whether AT1Rs on PVMs participate in the cerebrovascular effects of ANGII. To this end, we used BM chimeras to replace PVMs with PVMs lacking AT1Rs in WT mice (Atr1 $\left.{ }^{-/}\right)$(20, 21). We then assessed the cerebrovascular effects of ANGII "slow pressor" hypertension in WT mice with transplanted Atr1 ${ }^{-/}$BM.

In preparation for these studies, we first determined the extent and rate of replacement of PVMs by BM-derived monocytes using WT mice with transplanted $\mathrm{GFP}^{+} \mathrm{BM}$. After BM transplant, $\mathrm{GFP}^{+} \mathrm{CD} 2 \mathrm{O6}^{+}$cells with the characteristic morphology of PVMs were observed along pial and penetrating vessels (Figure 5A). By 14 weeks after the BM transplant, $78 \% \pm 2 \%$ of $\mathrm{CD}_{2} 26^{+}$cells were also $\mathrm{GFP}^{+}$, attesting to the high proportion of host PVMs replaced by donor PVMs (Figure 5B). Genomic DNA analysis confirmed that BM-derived cells were $A t 1 r^{-}$in WT mice engrafted with At1 ${ }^{-/}$marrow $\left(A t 1 r^{--} \rightarrow \mathrm{WT}\right)$, and $A t 1 r^{+/+}$in WT mice engrafted with WT marrow $(\mathrm{WT} \rightarrow \mathrm{WT})$ (Supplemental Figure 4A). The chimerism $\left(\right.$ At1 $\left.r^{\prime} \rightarrow \mathrm{WT}\right)$ was greater than 95\% (Supplemental Figure 4A). Since ANGII can influence monocyte mobilization and trafficking (31), we sought to rule out that At1r deletion or ANGII administration alters monocyte migration and homing to the perivascular space. To this end, we counted perivascular $\mathrm{CD}^{2} \mathrm{O6}^{+}$cells (PVMs) in the neocortex of $\mathrm{BM}$ chimeras with and without ANGII administration. No effect of At1r deletion or ANGII administration on PVM numbers was found (Supplemental Figure 4E).

CBF studies were performed 14 weeks after BM transplant, when the majority of PVMs had been replaced by the donor's macrophages (see above). In BM chimeras, ANGII administration elevated $\mathrm{BP}$ equally in $\mathrm{WT} \rightarrow \mathrm{WT}$ and $A t 1 r^{-} \rightarrow \mathrm{WT}$ chimeras (Figure $5 \mathrm{C}$ and Supplemental Figure 4C). WT mice engrafted with WT $\mathrm{BM}(\mathrm{WT} \rightarrow \mathrm{WT})$ exhibited normal cerebrovascular reactivity and attenuation by ANGII (Figure 5D), indicating that the irradiation and $\mathrm{BM}$ transplantation did not alter basic $\mathrm{CBF}$ responses and their susceptibility to ANGII. However, in At1r ${ }^{--} \rightarrow$ WT chimeras $\mathrm{CBF}$ responses to neural activation and $\mathrm{ACh}$ were markedly improved (Figure 5D). We then sought to assess the specificity of the rescue of the ANGII-induced cerebrovascular alterations in $A t 1 r^{-1} \rightarrow$ WT chimeras. We found that $A t 1 r^{-/} \rightarrow$ WT chimeras were protected from the cerebrovascular dysfunction induced by neocortical application of ANGII, but not of amyloid- $\beta$ (Figure 6, A and $\mathrm{B})$, a peptide that induces cerebrovascular dysfunction independently of AT1Rs (32). Therefore, AT1Rs in PVMs are required only for the neurovascular dysfunction induced by ANGII.

NOX2 in PVMs is required for the cerebrovascular effects of ANGII. Activation of AT1Rs leads to production of ROS derived from a NOX2-containing NADPH oxidase that has been implicated in the neurovascular dysfunction induced by ANGII slow pressor hypertension (10). Since macrophages are enriched in NOX2, in these experiments we sought to determine whether NOX2 in PVMs is responsible for the ANGII-induced neurovascular dys- 
A

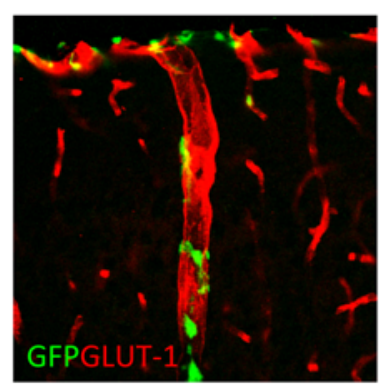

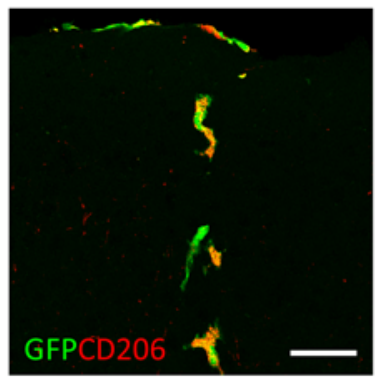

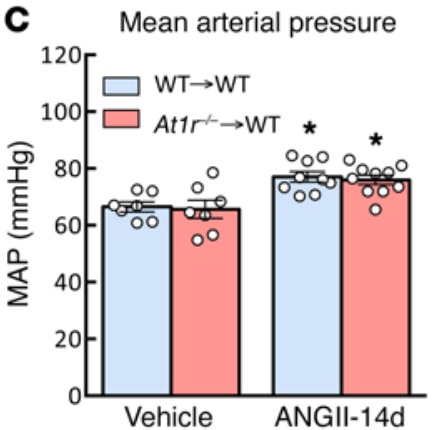

D

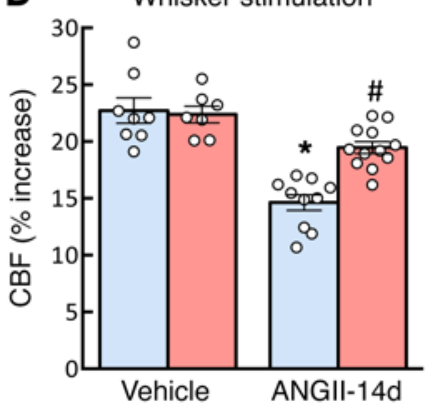

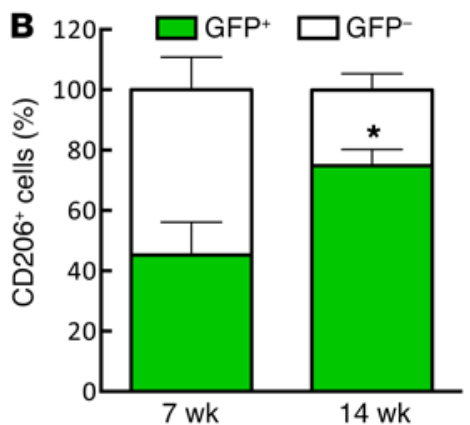

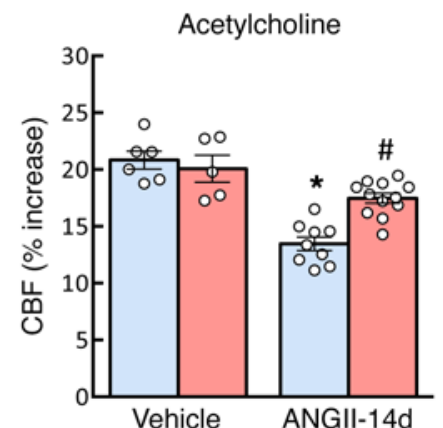

Figure 5. PVM At1r deletion counteracts the harmful cerebrovascular effects of ANGII slow pressor hypertension. (A) Fourteen weeks after transplant of GFP+ bone marrow in WT mice, GFP+ and CD206+ PVM surrounds GLUT-1+ vessels. Scale bar: $50 \mu \mathrm{m}$. (B) By 14 weeks after the transplant, approximately $80 \%$ of $\mathrm{CD}^{2} 26^{+}$cells in the somatosensory cortex are replaced by GFP+ bone marrow cells. ${ }^{*} P<0.05$ vs. 7 weeks group $\left[\chi^{2}(1)=7.78\right]$; $n=5$ per group. (C) ANCII increases mean arterial pressure equally in WT mice with transplanted WT bone marrow (WT $\rightarrow$ WT) or At1r ${ }^{-1-}$ marrow $\left(\right.$ At1r ${ }^{-1-}$ WT $) .{ }^{*} P<0.05$ vs. WT $\rightarrow$ WT-Veh and At1r ${ }^{-1-} \rightarrow$ WT-Veh; $n=7-10$ per group (2-way ANOVA and Bonferroni's test). (D) At1r deletion in PVMs does not affect the CBF responses to whisker stimulation or ACh, but counteracts the attenuation of both responses induced by ANGII. ${ }^{*} P<0.05$ vs. $\mathrm{WT} \rightarrow \mathrm{WT}-$ Veh and $A t 1 r^{-1-} \rightarrow \mathrm{WT}$-Veh; ${ }^{\#} P<0.05$ vs. WT $\rightarrow$ WT-ANGII; $n=7-12$ per group (2-way ANOVA and Bonferroni's test).

function. To this end, we generated $\mathrm{Nox}^{-/-} \rightarrow$ WT chimeras. First, we confirmed, by genomic DNA analysis in BM-derived cells, that, in Nox2 $2^{-/} \rightarrow$ WT chimeras, PVMs are Nox $2^{-/-}$(Supplemental Figure $4 \mathrm{~B})$. Fourteen weeks after BM transplant, ANGII minipumps were implanted, and cerebrovascular function was tested 2 weeks later. Nox2 deletion in BM cells (Nox $2^{--} \rightarrow$ WT chimeras) did not affect slow pressor hypertension (Supplemental Figure 4D) or baseline cerebrovascular reactivity (Figure 7A), but ameliorated the cerebrovascular dysfunction induced by ANGII in (Figure 7A), implicating NOX2 in PVMs in its mechanisms. However, the rescue of the dysfunction was not complete, possibly implicating ROS sources other than NOX2 (33).

Deletion of At1r or Nox 2 in PVMs attenuates ANGII-induced vascular oxidative stress. The cerebrovascular dysfunction induced by ANGII slow pressor hypertension is mediated by ROS $(10,16,17$, 34). ANGII increases vascular ROS production, and the increase is reversed by ROS scavengers, and is not observed in NOX2-null mice, implicating a NOX2-containing NADPH oxidase as a source of ROS (10). Because of their close apposition to the outer vessel wall and large capacity for ROS production, PVMs can be a powerful source of vascular oxidative stress. As illustrated in Figure 7B, ANGII induces an increase in ROS signal, assessed by hydroethidine microfluorography $(10,35)$ in PVMs. To test whether deletion of At1r or Nox2 in PVMs suppresses oxidative stress induced by ANGII, we used $A t 1 r^{--} \rightarrow$ WT and $N^{2} \times 2^{-/-} \rightarrow$ WT chimeras and
$\mathrm{WT} \rightarrow \mathrm{WT}$ chimeras as controls. In WT $\rightarrow$ WT mice, ANGII increases vascular ROS production $(10,35)$. In both $A t 1 r^{--} \rightarrow \mathrm{WT}$ and Nox $2^{-/} \rightarrow$ WT chimeras, the increase in ROS induced by ANGII was attenuated (Figure 7, C and D), indicating that PVMs through AT1Rs and NOX2 are responsible for the vascular oxidative stress induced by ANGII in the somatosensory cortex.

$P V M$ depletion ameliorates CBF dysfunction also in chronically hypertensive $\mathrm{BPH} / 2 \mathrm{~J}$ mice. A drawback of the slow pressor ANGII hypertension is that the elevation in $\mathrm{BP}$ is induced pharmacologically and is restricted to 2 weeks. To overcome these limitations, we also examined the role of PVMs in a model of spontaneous lifelong hypertension more representative of chronic hypertension in humans. To this end, we used $\mathrm{BPH} / 2 \mathrm{~J}$ mice, a mouse line developed from breeding of several strains of mice and selecting for the hypertension phenotype (36). BPH/2J mice exhibited elevated BP at 4 weeks of age (Supplemental Figure 5A), compared with normotensive controls, and the BP elevation remained stable until the mice were studied at 4-6 months of age. Because of their shortened lifespan (37), this age corresponds to middle age. At this time, levels of ANGII were elevated in plasma but not brain (Supplemental Figure 5, B and C). The BBB permeability to FITC-dextran was increased (Figure 8A). The increase in CBF induced by functional hyperemia or ACh was attenuated, whereas the CBF response to adenosine was normal (Figure $8 \mathrm{~B}$ and Supplemental Table 1), attesting to the integrity of the vasorelaxing function of 
A

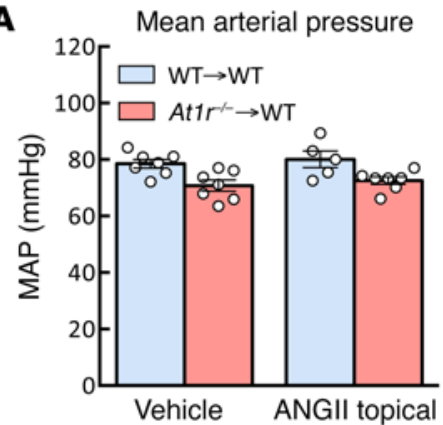

B

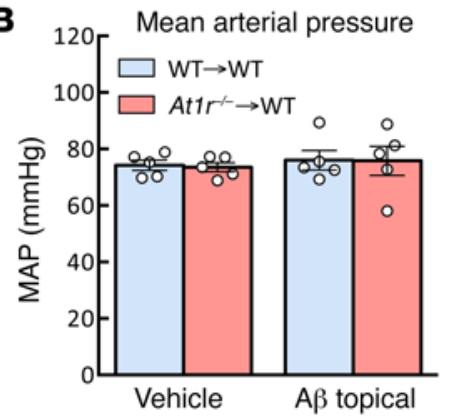

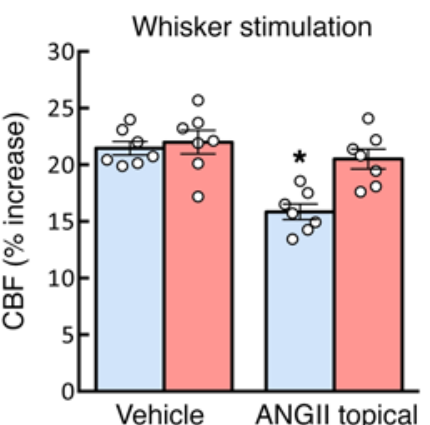
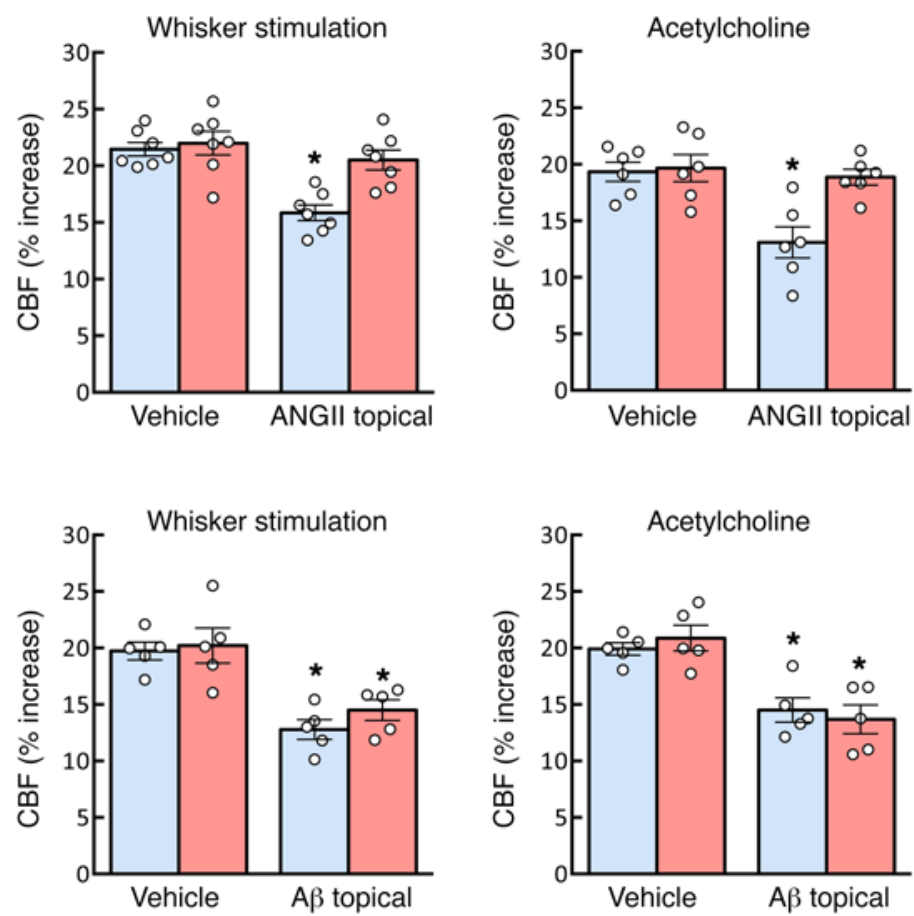

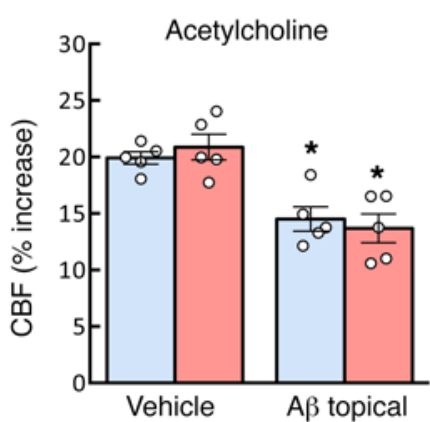

Figure 6. At1r deletion in PVMs rescues the neurovascular dysfunction induced by topical neocortical application of ANGII but not amyloid- $\beta$. (A) At1r/- $\rightarrow$ WT chimeras are protected from the cerebrovascular effects induced by topical neocortical application of ANGIl. ${ }^{*} P<0.05$ vs. WT $\rightarrow$ WT-Veh and At1r ${ }^{\prime-} \rightarrow$ WT-Veh; $n=5-7$ per group (2-way repeated-measures ANOVA and Bonferroni's test). (B) The attenuation of the CBF responses induced by topical application of amyloid- $\beta$ (AB), an effect not dependent on AT1Rs, is not affected. This finding attests to the specificity of the effect of PVM At1r deletion on the cerebrovascular dysfunction induced by ANGII. ${ }^{*} P<0.05$ vs. WT $\rightarrow$ WT-Veh and $A t 1 r^{-1-} \rightarrow$ WT-Veh (2-way repeated-measures ANOVA and Bonferroni's test).

the smooth muscle. The CBF dysfunction was counteracted by neocortical application of the AT1R antagonist losartan or the ROS scavenger MnTBAP (Figure 8B), indicating that the neurovascular alterations are mediated by AT1Rs and ROS. CLOD administration (i.c.v.) in $\mathrm{BPH} / 2 \mathrm{~J}$ mice depleted PVMs in both cortex and hippocampus (Supplemental Figure 5, D and E), and rescued the response to both whisker stimulation and ACh (Figure 8D). These data implicate PVMs also in the cerebrovascular dysfunction occurring in a model of lifelong spontaneous hypertension.

$P V M$ depletion improves cognitive function in $\mathrm{BPH} / 2 \mathrm{~J}$ mice with chronic hypertension. Finally, we sought to determine whether the rescue of cerebrovascular dysfunction afforded by PVM depletion leads to an improvement in the cognitive deficits associated with hypertension. In this study, we elected to use middle-aged $\mathrm{BPH} / 2 \mathrm{~J}$ mice, a model of spontaneous lifelong hypertension resulting in well-documented and reproducible cognitive deficits (38). We did not assess cognitive function in ANGII-treated mice because higher concentrations of ANGII and longer exposures are needed to observe cognitive deficits (4 weeks) $(39,40)$. Such longer administration times are not compatible with the time course of PVM depletion by CLOD, since PVMs begin to repopulate the perivascular space at this time (27). To examine the role of PVMs in the cognitive deficits in $\mathrm{BPH} / 2 \mathrm{~J}$ mice, we first used the novel object recognition task, a test of recognition memory that relies on the propensity of mice to explore novel objects more than familiar ones (41). Normotensive mice presented with an object to which they were previously exposed and a novel one spent more time exploring the novel object (Figure 9A). In contrast, BPH/2J mice spent equal time exploring the novel and the familiar object, reflecting a deficit in recognition memory (Figure 9A). The effect was observed both 1 and 24 hours after the familiarization trial (Supplemental Figure 5, F and G). CLOD treatment did not affect the performance of the normotensive controls and the total exploration time in both groups, but greatly ameliorated the memory deficit in $\mathrm{BPH} / 2 \mathrm{~J}$ mice (Figure 9A).

To provide additional evidence for the involvement of PVMs in the cognitive deficits induced by chronic hypertension, we then used the Barnes maze. This test is similar to the water maze navigation task in that it examines the ability of the mouse to learn and remember the location of a target zone (spatial learning and memory) (42). However, the Barnes maze has the advantage of avoiding the strong aversive stimulus of swimming that may affect performance (43). The mouse is placed on a circular platform with holes at its outer edge, 1 of which is the correct escape hole. Within a few trials a normal mouse will accurately identify the escape hole, avoiding the incorrect holes. The test is repeated daily for 3 days to test the ability of the mouse to retain the information, once the location of the escape hole was learned. Spatial memory is assessed by measurement of both the time spent to find the escape hole (primary latency) and the distance traveled to do so (44). Both $\mathrm{BPH} / 2 \mathrm{~J}$ and normotensive controls showed spatial learning over the 3-day test, as indicated by the reduced primary latency and distance traveled (Figure 9, $\mathrm{B}$ and $\mathrm{C})$. However, both primary latency and distance traveled were significantly higher in $\mathrm{BPH} / 2 \mathrm{~J}$ mice compared with normotensive mice, reflecting spatial memory impairment (Figure 9, B 
A

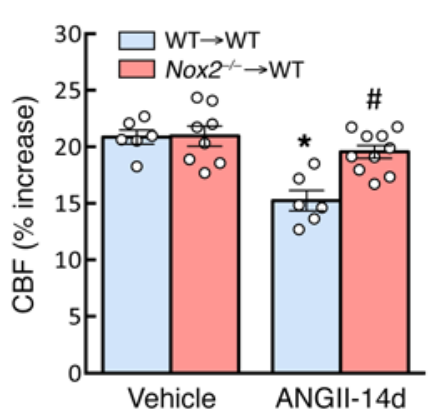

Acetylcholine

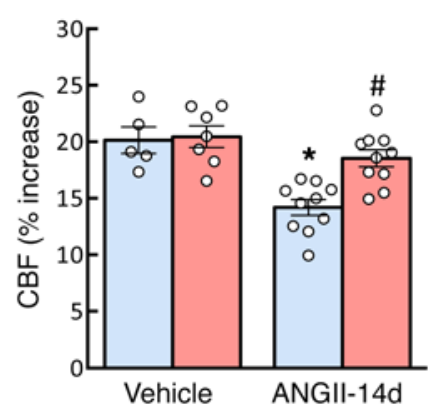

B
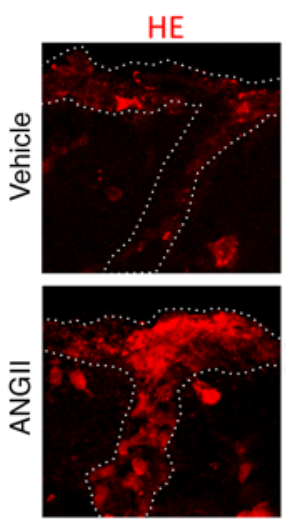
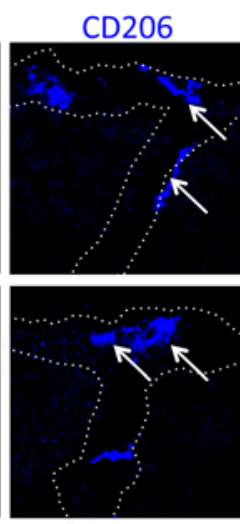
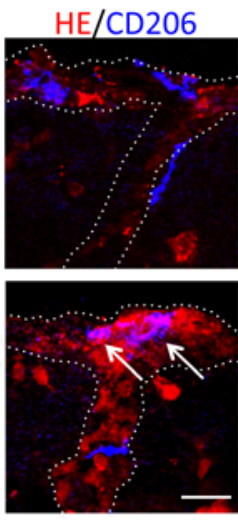

C
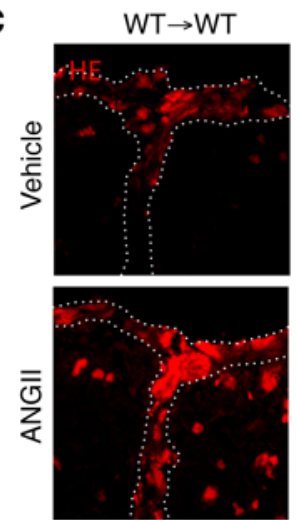
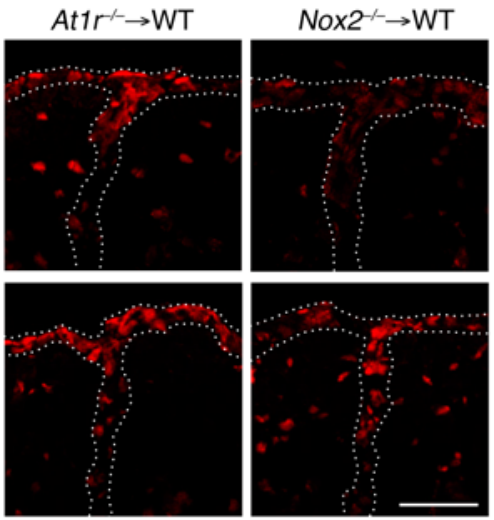

D

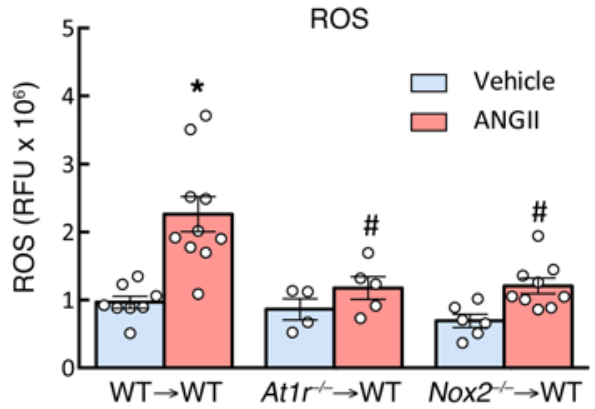

Figure 7. NOX2 in PVMs is required for the cerebrovascular effects of ANGII slow pressor hypertension. (A) Nox2 deletion in PVMs does not affect baseline cerebrovascular responses, but counteracts the cerebrovascular dysfunction induced by ANGIl (Nox2 ${ }^{-1-} \rightarrow$ WT chimeras). ${ }^{*} P<0.05$ vs. WT $\rightarrow$ WT-Veh and Nox2 ${ }^{-1} \rightarrow$ WT-Veh; ${ }^{~} P<0.05$ vs. WT $\rightarrow$ WT-ANGIl; $n=5-10$ per group (2-way ANOVA and Bonferroni's test). (B) ANGll markedly increases ROS production in $\mathrm{CD}_{206}{ }^{+}$cells (PVMs) assessed by dihydroethidine (DHE) microfluorography. Scale bar: $25 \mu \mathrm{m}$. (C and D) Deletion of At1r or Nox2 in PVMs attenuates the neurovascular oxidative stress induced by slow pressor ANCII in the neocortex. Scale bar: $50 \mu \mathrm{m} .{ }^{*} P<0.05$ vs. WT $\rightarrow$ WT-Veh, At1r ${ }^{-1-} \rightarrow$ WT-Veh, and Nox2 $2^{-1} \rightarrow$ WT-Veh; ${ }^{\#} P<0.05$ vs. WT $\rightarrow$ WT-ANGII; $n=4-10$ per group (2-way ANOVA and Bonferroni's test).

and C). PVM depletion by CLOD did not affect the performance of the normotensive controls, but significantly improved the cognitive performance of the $\mathrm{BPH} / 2 \mathrm{~J}$ mice (Figure 9C).

\section{Discussion}

We tested the hypothesis that PVMs are involved in the cerebrovascular and cognitive dysfunction induced by hypertension. First, we showed that in the slow pressor model of hypertension ANGII crosses the BBB and reaches PVMs in the perivascular space, demonstrating that these cells have access to circulating ANGII. Next, we demonstrated that PVM depletion using CLOD does not alter baseline cerebrovascular responses, but rescues the neurovascular dysfunction induced by slow pressor ANGII administration, or observed in a genetic model of lifelong hypertension. Then, we asked whether AT1Rs and NOX2 in PVM are involved in the neurovascular dysfunction induced by ANGII. Using BM chimeras we provided evidence that replacement of WT PVMs with PVMs lacking AT1Rs or NOX2 abrogates the vascular oxidative stress induced by ANGII and counteracts the associated neurovascular dysfunction. The effect was specific for the neurovascular dysfunction of ANGII, since At1r deletion in PVMs did not rescue the dysfunction induced by the amyloid- $\beta$ peptide. Finally, we tested whether the rescue of CBF dysfunction induced in hyper- tension was associated with cognitive improvement. We found that PVM depletion completely reverses the deficits in both recognition and spatial memory observed in $\mathrm{BPH} / 2 \mathrm{~J}$ mice with lifelong hypertension. These novel observations unveil an obligatory role of brain PVM in the neurovascular and cognitive dysfunction associated with hypertension.

PVMs are tissue macrophages that reside constitutively in selected organs, such as brain and skin (45), wherein they subserve a wide variety of homeostatic functions, including immune surveillance, defense from infections, and neutrophil trafficking $(46,47)$. In brain, they are distinct from other perivascular cells and microglia for their morphology, immune phenotype, and propensity to phagocytosis $(18,25)$. Brain PVMs are thought to be beneficial in models of Alzheimer disease by removing amyloid- $\beta$ peptides from the perivascular space and preventing amyloid accumulation in cerebral blood vessels (amyloid angiopathy) $(48,49)$. On the other hand, hypothalamic neurohumoral signaling by PVMs across the BBB may be deleterious by promoting inflammation and sympathetic activation in models of fever or myocardial infarction, respectively $(28,29)$, and may contribute to hypertensive cerebrovascular remodeling (50). In the present study we discovered that PVMs play a key role in the cerebrovascular dysfunction of hypertension. We found that 
A Mean arterial pressure
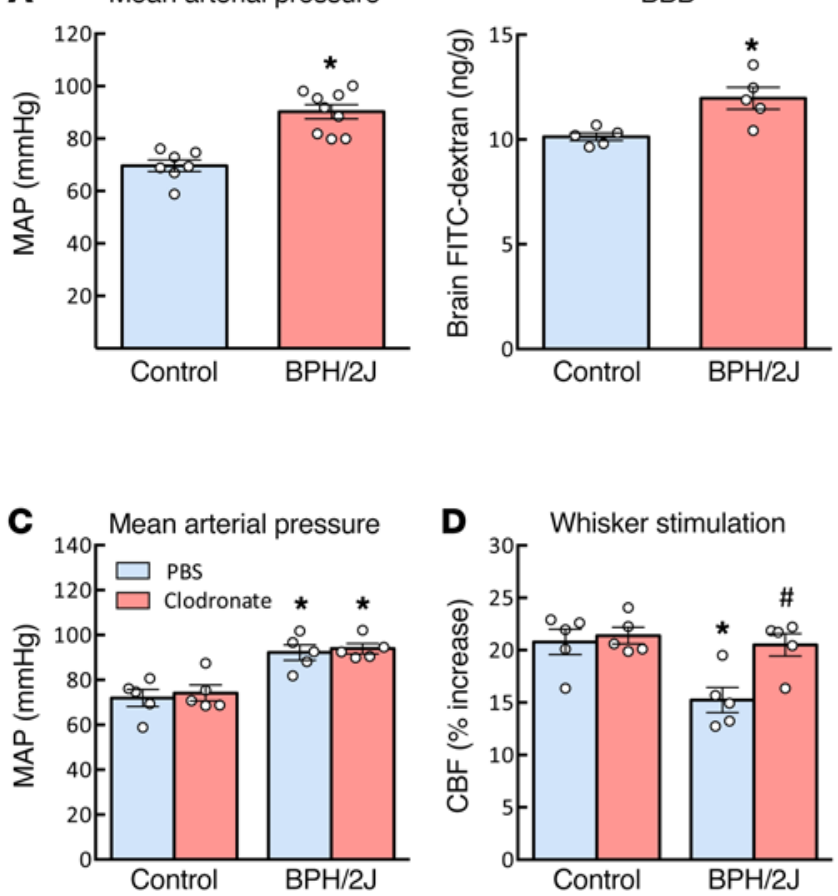

B

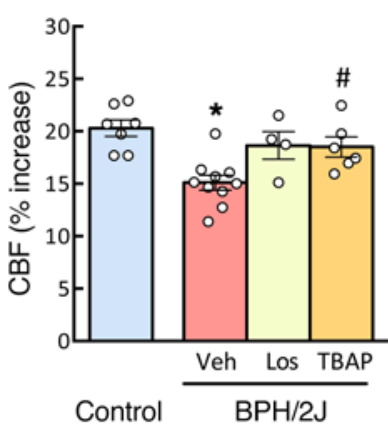

Acetylcholine

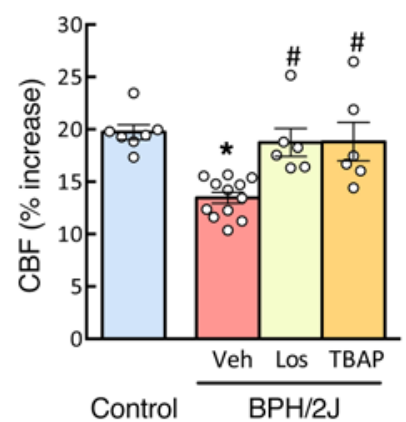

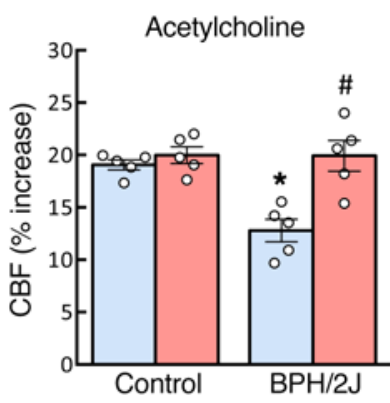

Figure 8. PVMs mediate cerebrovascular dysfunction in chronically hypertensive BPH/2J mice. (A) Mean arterial pressure and BBB permeability to FITC-dextran are increased in BPH/2 J mice. ${ }^{*} P<0.05$ vs. control; ${ }^{P} P<0.05$ vs. Veh; $n=5-9$ (Student's $t$ test). (B) Antagonism of AT1Rs with losartan or ROS scavenging with MnTBAP counteracts the neurovascular dysfunction in $\mathrm{BPH} / 2$ ) mice. ${ }^{*} P<0.05 \mathrm{vs}$. control; $n=4-7$ per group (1-way ANOVA and Tukey's test). (C and $\mathbf{D})$ CLOD has no effect on the mean arterial pressure but completely reverses the attenuation in CBF response to whisker stimulation and $\mathrm{ACh}$ in $\mathrm{BPH} / 2$ ) mice. ${ }^{*} P<0.05$ vs. PBS-control and CLOD-control; ${ }^{~} P<0.05$ vs. PBS-BPH/2); $n=5$ per group (2-way ANOVA and Bonferroni's test).

ANGII hypertension or chronic elevation of $\mathrm{BP}$ in $\mathrm{BPH} / 2 \mathrm{~J}$ mice does not increase the number of PVMs or alter their homing from the circulation to the perivascular space. However, PVMs become the target of circulating ANGII, leading to engagement of AT1Rs, NOX2-dependent ROS formation, and neurovascular and cognitive dysfunction. Our data implicate brain PVMs in the cognitive deficits induced by hypertension, and identify resident brain PVMs as a novel constituent of the NVU highly relevant to brain pathophysiology.

The NVU, a construct originally introduced to highlight the close developmental, structural, and functional relationships among neurons, glia, and cerebrovascular cells, is essential for the maintenance of the homeostasis of the cerebral microenvironment (6). Neurovascular coupling represents the prototypical homeostatic response of the NVU implemented by coordinated signaling among neurons, astrocytes, smooth muscle cells, pericytes, and endothelial cells, and involving a wide variety of mediators (7). Although the specific role that NVU cells, such as astrocytes and pericytes, play in neurovascular coupling is still being debated $(51,52)$, it is well established that neurovascular dysfunction leads to neuronal dysfunction in particularly susceptible cerebrocortical and hippocampal neuronal circuits, resulting in cognitive impairment (12). The present results expand the concept of NVU by highlighting a novel role of PVMs as a critical cell for neurovascular disruption in hypertension. Our data suggest that PVMs, while serving vital homeostatic functions in the normal state, become the target of neurovascular inflammatory signaling leading to ROS production, vascular dysfunction, and cognitive deficits. However, the molecular interactions of PVMs with cells of the NVU and their role in the neurovascular dysfunction and BBB alteration remain to be defined.

It has long been known that vascular oxidative stress plays a key role in the cerebrovascular alterations induced by hypertension, but the vascular cells specifically involved in ROS generation have not been identified (2). Cerebral endothelial cells express AT1Rs and NOX2 (11), but their potential for pathological ROS production is relatively limited compared with that of macrophages; thus they are unlikely to be the sole source of oxidative stress. Similarly, smooth muscle cells (a) are not a major source of ROS in models of cerebrovascular oxidative stress (53), (b) their vasomotor function is not impaired in ANGII hypertension (10, 15, $34,35)$, and (c) their AT1R expression level in cerebral arterioles is low (11). Our findings demonstrate that PVMs are potent ROS generators in slow pressor and lifelong hypertension. Thus, PVMs emerge as a novel source of vascular oxidative stress in the cerebral microcirculation with a profound impact on neurovascular regulation and cognitive function. However, the functional rescue afforded by Nox 2 deletion in PVMs is not complete. Although a contribution of the $20 \%-30 \%$ of PVMs not replaced by the transplant of $\mathrm{Nox}^{-/-} \mathrm{BM}$ cannot be ruled out, it is also possible that other cellular sources of ROS are also at play.

We also found that ANGII is able to cross the $\mathrm{BBB}$ and reach the perivascular space. BBB permeability tended to increase before the onset of hypertension, like the cerebrovascular dysfunction, which is first observed 7 days after ANGII administration (54). This is not surprising, since the neurovascular dysfunc- 
A

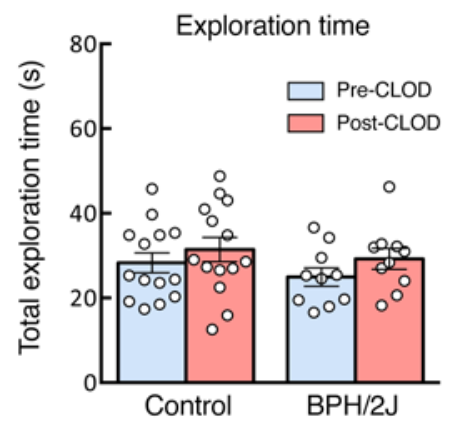

B

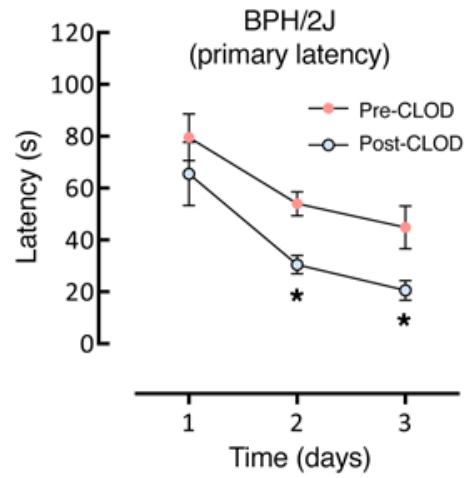

C

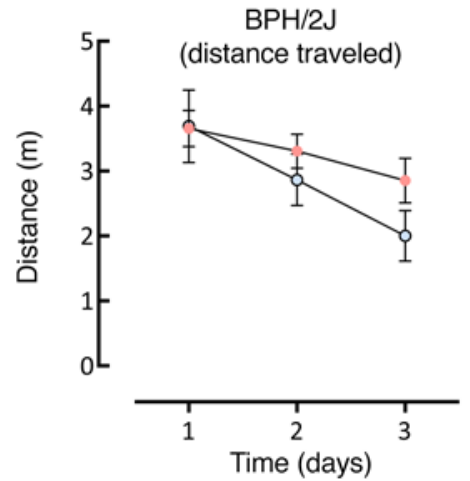

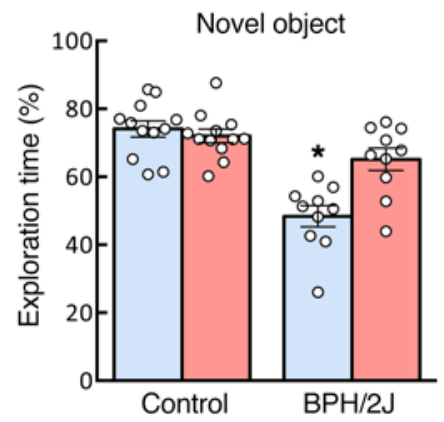

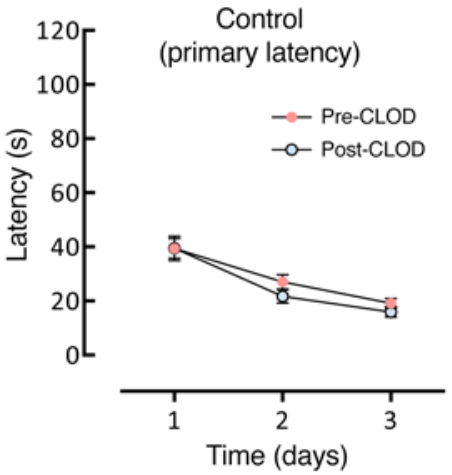

D Control

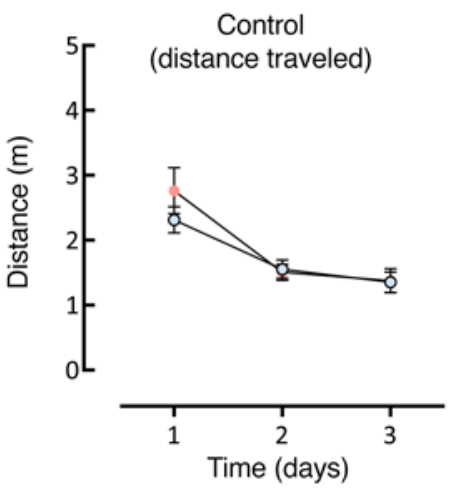

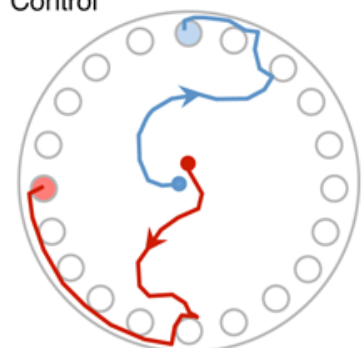

$\mathrm{BPH} / 2 \mathrm{~J}$

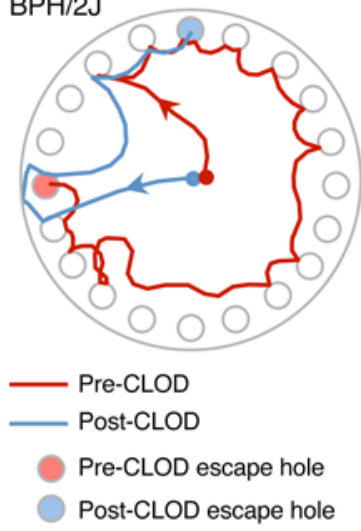

Figure 9. PVMs mediate cognitive dysfunction in chronically hypertensive BPH/2J mice. (A) PVM depletion by CLOD rescues the recognition memory deficits assessed by the novel object recognition test in $\mathrm{BPH} / 2$ ) mice. ${ }^{*} P<0.05$ vs. pre-CLOD-control and post-CLOD-control; $n=10-12$ per group (2-way repeated-measures ANOVA and Bonferroni's test). (B) PVM depletion does not affect the spatial memory assessed by Barnes maze test in control mice but rescues the deficits observed in BPH/2) mice. ${ }^{*} P<0.05$ vs. pre-CLOD-BPH/2); $n=15-20$ per group (2-way repeated-measures ANOVA and Bonferroni's test). (C) CLOD tends to reduce the distance traveled in $\mathrm{BPH} / 2$ J mice, but the effect does not reach statistical significance. $P>0.05$ vs. pre-CLOD-BPH/2J; $n=15-20$ per group (2-way repeated-measures ANOVA and Bonferroni's test). (D) Representative tracks for control and BPH/2) mice on acquisition day 3 before and after vehicle or CLOD injection.

tion and the BBB dysfunction are likely to be expressions of the same pathogenic process (2). Whether ANGII crosses the BBB has been a long-standing open question $(55,56)$. Although early studies described BBB opening by sudden increases in BP induced by high concentrations of ANGII or other pressor agents, these investigations used massive increases in BP that caused acute vascular damage and total vasoparalysis (57). Subsequent studies with more moderate and long-lasting increases in BP or in models of chronic hypertension demonstrated in some cases regional ANGII-dependent increases in BBB permeability (e.g., ref. 55), but it remained unclear whether ANGII itself was able to cross the $\mathrm{BBB}$ in slow pressor ANGII hypertension. Using markers of differ- ent sizes we were able to clearly detect a size-dependent opening of the BBB in ANGII slow pressor hypertension. In our studies with acute hypertension, in which the rise in BP was modest and comparable to that in slow pressure hypertension, we did not observe increased BBB permeability, attesting to the fact that the elevation in $\mathrm{BP}$ is not driving the BBB alteration. This conclusion is reinforced by the experiments in which the BBB dysfunction was not observed if BP was elevated for 2 weeks by phenylephrine. Experiments with biotinylated ANGII demonstrated more directly that ANGII reaches the perivascular space and enters in contact with PVMs. Since brain levels were not increased, ANGII may not enter the brain parenchyma in massive amounts but, as we showed, 


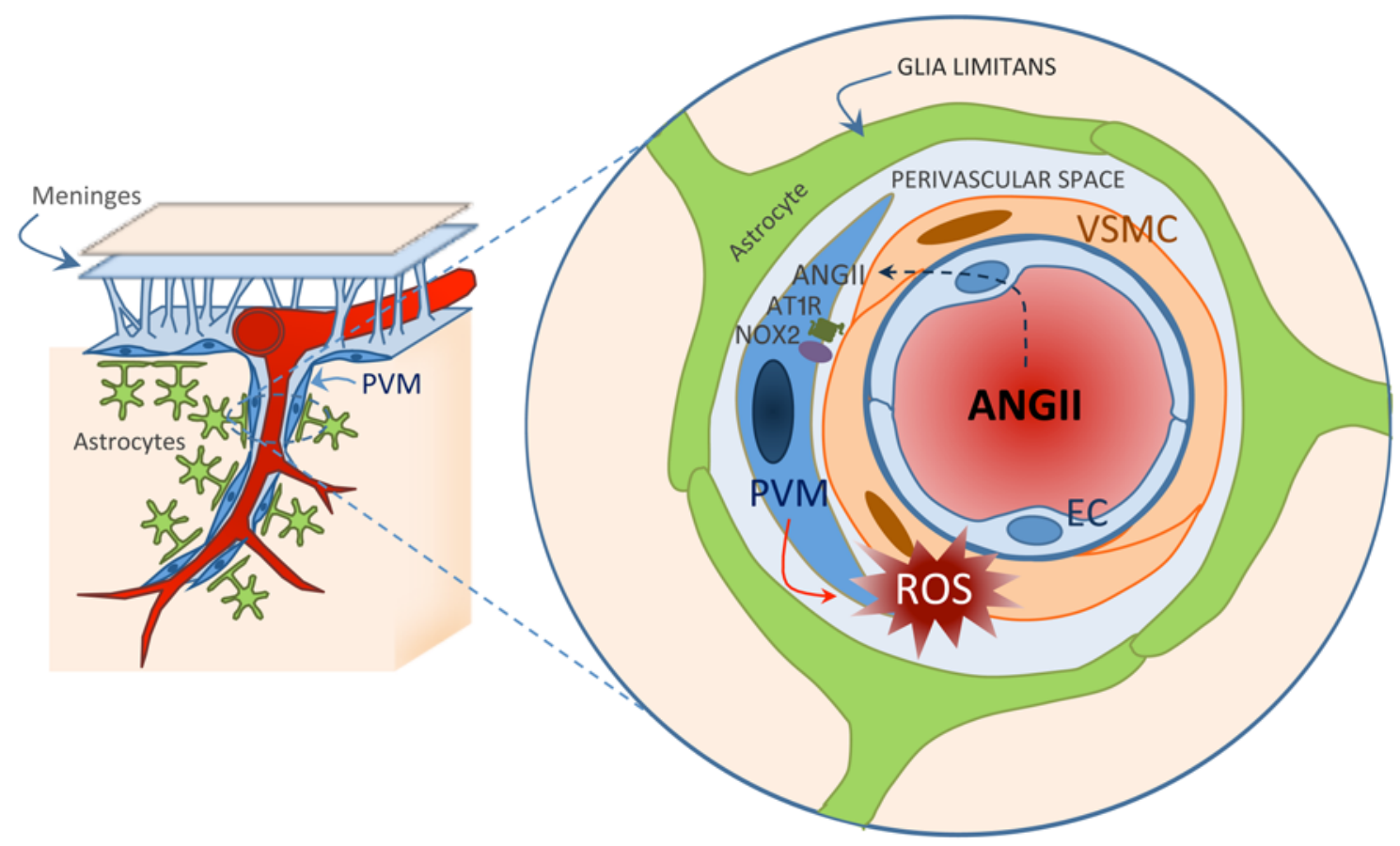

Figure 10. Potential mechanisms by which ANGII hypertension leads to neurovascular dysfunction underlying cognitive deficits. The left side of the figure illustrates a pial arteriole giving off a branch penetrating into the brain parenchyma and surrounded by astrocytic end-feet forming the glia limitans and by PVMs. As illustrated in the enlargement on the right, circulating ANGIl reaches the perivascular space through a breach of the BBB and acts on ANGII type 1 receptors (AT1R) on PVMs, resulting in the activation of NOX2 and ROS production. Oxidative stress, in turn, leads to neurovascular dysfunction. PVM, perivascular macrophage; EC, endothelial cell; VSMC, vascular smooth muscle cell.

remains largely confined to the perivascular space, wherein it activates ROS production in PVMs. The mechanisms of the increase in BBB permeability by ANGII remain to be established. Ongoing studies are examining the role of AT1Rs in endothelial cells as the first step in the BBB opening that allows the entry of ANGII in the perivascular space, enabling PVM activation.

In the present study we investigated $\mathrm{BPH} / 2 \mathrm{~J}$ mice, a mouse line not previously used in cerebrovascular research. $\mathrm{BPH} / 2 \mathrm{~J}$ mice are a model of lifelong hypertension on a genetic basis and manifest some of the cardinal features of essential hypertension, i.e., neurohumoral dysfunction, sympathetic activation, cardiac hypertrophy, and kidney atrophy $(58,59)$. Due to long-lasting hypertension, $\mathrm{BPH} / 2 \mathrm{~J}$ mice are valuable in hypertension research, akin to spontaneously hypertensive rats. However, it was not known whether these mice also exhibited the cerebrovascular alterations caused by chronic hypertension. We found that BPH/2J mice exhibit cerebrovascular impairment as well as cognitive dysfunction. The CBF dysfunction was counteracted by neocortical superfusion with the AT1R antagonist losartan or the ROS scavenger MnTBAP, implicating AT1Rs and ROS in its mechanisms. After establishing the occurrence of cerebrovascular dysfunction in this model, we proceeded to investigate the role of PVMs. We found that PVM depletion counteracts the neurovascular dysfunction as well as the cognitive deficits in $\mathrm{BPH} / 2 \mathrm{~J}$ mice, providing evidence for a central role of PVMs in the cerebrovascular and cognitive dysfunction also in a nonpharmacological model of lifelong hypertension. Because of the complex genetic background of $\mathrm{BPH} / 2 \mathrm{~J}$ mice, we could not perform $\mathrm{BM}$ chimera experiments to pinpoint the involvement of AT1Rs and NOX2. At1r-- BM and Nox2 $2^{-}$BM are on a C57BL/6 background, and in preliminary experiments $\mathrm{BPH} / 2 \mathrm{~J}$ mice with transplanted $\mathrm{C} 57 \mathrm{BL} / 6 \mathrm{BM}$ developed graft versus host disease (not shown). Nevertheless, considering that $\mathrm{BPH} / 2 \mathrm{~J}$ mice have increased BBB permeability and increased levels of circulating ANGII, and that their cerebrovascular dysfunction depends on PVMs, AT1Rs, and ROS, it is reasonable to conclude that AT1Rs and NOX2 in PVMs are involved. The convergence of the findings in ANGII slow pressor hypertension with those in $\mathrm{BPH} / 2 \mathrm{~J}$ mice provides strong evidence for a central role of PVMs in the neurovascular and cognitive dysfunction produced by hypertension.

Increasing evidence indicates that adaptive immunity and innate immunity play a key role in the pathogenesis of hypertension and in the associated end-organ damage (60). Pioneering studies in lymphocyte-deficient mice (Rag1 ${ }^{-/}$mice) established that T lymphocytes are required for the BP elevations in several hypertension models (61). The effect is mediated by infiltration of the vascular wall by $\mathrm{T}$ lymphocytes and macrophages leading to production of deleterious cytokines, such as IL-17 and TNF- $\alpha$, which, in turn, contribute to the increased vascular resistance driving the hypertension, as well as end-organ damage (61). In addition, innate immune cells, particularly macrophages, have been implicated in vascular remodeling and atherosclerosis through the production of ROS and cytokines (19). Our findings implicate, for the first time to our knowledge, a distinct population of innate immune cells, brain-resident PVMs, in the neurovascular and neurocognitive dysfunction associated with hypertension (Figure 10). PVMs are not involved 
in the elevation of $\mathrm{BP}$ induced by ANGII or in $\mathrm{BPH} / 2 \mathrm{~J}$ mice, but are a major source of vascular ROS mediating the neurovascular dysfunction, highlighting their specific involvement in the brain vasculature. Therapeutic strategies targeting PVMs may provide a new approach to counteract the harmful effects of cerebrovascular oxidative stress and may help ameliorate the end-organ damage to the brain in the setting of hypertension and related cerebrovascular pathologies. Although plasma levels of ANGII are not consistently elevated in essential hypertension (13), activation of tissue-specific renin-angiotensin systems, including in brain and blood vessels, is thought to play a role (62). Neurovascular coupling and BBB are altered in essential hypertension (63), and observational studies indicate that ANGII antagonists can protect the brain from hypertension-induced cognitive dysfunction (64). However, evidence from randomized clinical trials that treatment of hypertension reduces dementia risk and that there is a class-specific effect of drugs targeting ANGII and its receptors is lacking (3). Studies addressing this issue are eagerly awaited.

Conclusions. We tested the hypothesis that PVMs are involved in the neurovascular and cognitive dysfunction induced by hypertension. After establishing that ANGII crosses the BBB to reach PVMs in the perivascular space, we demonstrated that depletion of PVMs rescues the neurovascular and cognitive dysfunction associated with hypertension. In a model of slow pressor ANGII hypertension, BM chimera experiments demonstrated that AT1Rs and NOX2 in PVMs are needed for the vascular oxidative stress and neurovascular dysfunction. The findings unveil a previously unappreciated role of PVMs in the full expression of the neurovascular and neurocognitive dysfunction of hypertension, and identify PVMs as a new cellular component of the NVU of critical importance for neurovascular diseases associated with vascular oxidative stress.

\section{Methods}

General surgical procedures. Studies were conducted, according to ARRIVE guidelines (https://www.nc3rs.org.uk/arrive-guidelines), in male C57BL/6 mice (age 3-5 months, weight 25-30 g; JAX, The Jackson Laboratory), in male $\mathrm{BPH} / 2 \mathrm{~J}$ mice (age 5 months, weight 20-25 g; JAX stock 003005), and in their respective controls, male BPN/3J mice (age 5 months, weight 25-30 g; JAX stock 003004).

$B M$ transplant. As previously described (65), whole-body irradiation was performed on 7-week-old male C57BL/6 mice with a lethal dose of $9.5 \mathrm{~Gy}$ of $\gamma$ radiation using a ${ }^{137} \mathrm{Cs}$ source (Nordion Gammacell 40 Exactor). Eighteen hours later, BM cells $\left(2 \times 10^{6}\right.$, i.v. $)$ isolated from donor B6.129P2-Agtr1a ${ }^{-/-}\left(\right.$At1r $^{-/}$, JAX stock 002682) and B6.129S-Cybbtm1Din/J mice (Nox2 ${ }^{-/}$, JAX stock 002365) were transplanted in irradiated mice. Mice with transplanted BM cells were housed in cages with sulfamethoxazole $(0.12 \%$, wt/vol) and trimethoprim $(0.024 \%)$ antibiotics added to drinking water for the first 2 weeks. Reconstitution of BM cells was verified 5 weeks after irradiation by testing of the percentage of positive At1r or Nox2 genomic DNA in isolated blood leukocytes (65). Reference primer sequences were as follows: m_Icam1_prom.3, 5'-GGACTCACCTGCTGGTCTCT-3', and m_Icam1_prom.4, 5'-GAACGAGGGCTTCGGTATTT-3'. Target primer sequences were as follows: $\mathrm{m}_{-} A g$ tr1a_gt 1, 5'-TTTGCGCTTTTCATTACGAGTCC-3'; m_Agtr1a_gt_2, 5'-GAAGCCCAGGATGTTCTTGGT-3'; m_Cybb_gt_1, 5'-CTGCT-
CACCAGCCTCTCTCTA-3'; and m_Cybb_gt_2, 5'-CTGGAACCCCTGAGAAAGGAG-3' (Invitrogen). Quantitative RT-PCR was conducted with $20 \mathrm{ng}$ of DNA, in duplicate $15-\mu l$ reactions using the Maxima SYBR Green/ROX qPCR Master Mix (2×) (Thermo Scientific). A PCR cycling protocol consisting of 15 seconds at $95^{\circ} \mathrm{C}$ and 1 minute at $60^{\circ} \mathrm{C}$ for 45 cycles was used for quantification. At1r or Nox 2 relative expression levels were calculated by $2^{-\Delta \Delta \mathrm{CT}}$ method (66). For histological studies, GFP-transgenic mice (JAX stock 006567) were used as WT BM donors, and $\mathrm{GFP}^{+}$(WT) BM was transplanted in irradiated WT mice.

Intracerebroventricular injection of liposomes. To deplete cerebral $\mathrm{CD}^{2} \mathrm{O6}^{+}$macrophages, clodronate- or PBS-loaded liposomes were prepared as previously described (27). Under isoflurane anesthesia, $10 \mu \mathrm{l}$ of clodronate-liposomes $(7 \mathrm{mg} / \mathrm{ml})$ and PBS-liposomes (vehicle) were slowly injected in cerebral ventricles (i.c.v.) with a glass micropipette through a burr hole drilled on the left parietal bone (coordinates: $0.2 \mathrm{~mm}$ posterior to bregma $1.0 \mathrm{~mm}$ lateral from midline, 2.3 $\mathrm{mm}$ below the brain surface).

Intracerebroventricular injection of dextran. Under isoflurane anesthesia, $5 \mu$ of a solution containing $5 \%$ of fluorescein-labeled dextran (FITC-dextran, 10,000 MW, lysine fixable, Molecular Probes, Fluoro-Emerald, D-1820) dissolved in saline was injected into the left lateral ventricle at the same coordinates used for liposome injections. Four hours later mice were anesthetized with sodium pentobarbital $(120 \mathrm{mg} / \mathrm{kg}$, i.p.) and transcardially perfused as described below.

Osmotic minipump implantation. Osmotic minipumps containing vehicle (saline) or ANGII (600 ng/ $\mathrm{kg} / \mathrm{min}$ ) were implanted s.c. under isoflurane anesthesia. Systolic BP was monitored in awake mice using tail-cuff plethysmography (35). Fourteen days later, mice were anesthetized and instrumented for assessment of cerebrovascular reactivity as described below.

General surgical procedures for CBF studies. Mice were anesthetized with isoflurane (induction, $5 \%$; maintenance, $2 \%$ ) and artificially ventilated with a mixture of $\mathrm{N}_{2}$ and $\mathrm{O}_{2}$ (53). One of the femoral arteries was cannulated for recording mean arterial pressure (MAP) and collecting blood samples. Rectal temperature was maintained at $37^{\circ} \mathrm{C}$. End tidal $\mathrm{CO}_{2}$, monitored by a $\mathrm{CO}_{2}$ analyzer (Capstar-100, CWE Inc.), was maintained at $2.6 \%-2.7 \%$ to provide a $\mathrm{pCO}_{2}$ of $30-40 \mathrm{mmHg}$ and a pH of 7.3-7.4 (67). After surgery, isoflurane was discontinued and anesthesia was maintained with urethane $(750 \mathrm{mg} / \mathrm{kg}$, i.p.) and chloralose $(50 \mathrm{mg} / \mathrm{kg}$, i.p.). Throughout the experiment the level of anesthesia was monitored by testing of motor responses to tail pinch. As in previous studies (54), mean BP was lowered by anesthesia, but still in the autoregulated range for $\mathrm{CBF}$.

Monitoring $C B F$. A small craniotomy $(2 \times 2 \mathrm{~mm})$ was performed to expose the parietal cortex, the dura was removed, and the site was superfused with Ringer's solution $\left(37^{\circ} \mathrm{C}\right.$; $\mathrm{pH}$ 7.3-7.4) (67). CBF was continuously monitored at the site of superfusion with a laser-Doppler probe (Perimed) positioned stereotaxically on the cortical surface and connected to a data acquisition system (PowerLab). CBF values were expressed as percentage increases relative to the resting level.

Experimental protocol for CBF experiments. After MAP and blood gases were stable, CBF responses were recorded. The whisker-barrel cortex was activated for 60 seconds by stroking of the contralateral vibrissae, and the evoked changes in CBF were recorded. ACh $(10 \mu \mathrm{M}$; Sigma-Aldrich) or adenosine ( $400 \mu \mathrm{M}$; Sigma-Aldrich) was superfused on the exposed neocortex for 5 minutes. In some studies, CBF 
responses were tested before and after 30 minutes of superfusion with amyloid- $\beta(5 \mu \mathrm{M})(32)$ and ANGII $(500 \mathrm{nM})(30)$, or after 30 minutes of i.v. infusion of ANGII (250 ng/ $\mathrm{kg} / \mathrm{min})$ (11). In control and $\mathrm{BPH} / 2 \mathrm{~J}$ mice, $\mathrm{CBF}$ responses were tested before and after 30 minutes of superfusion with the AT1R antagonist losartan $(5 \mu \mathrm{M})$ or the ROS scavenger $\operatorname{MnTBAP}(100 \mu \mathrm{M})(10,35)$. In chimeric mice, CBF experiments were performed 14 weeks after the BM transplant when most of the host PVMs have been replaced by donor macrophages.

Novel object recognition test. The novel object recognition test (NOR) task was conducted in a plastic box measuring $29 \mathrm{~cm} \times 47 \mathrm{~cm}$ $\times 30 \mathrm{~cm}$ high. Stimuli consisted of plastic objects that varied in color and shape but had similar size. A video camera was used to record the testing session for offline analysis. Mice were acclimated to the testing room and chamber for 1 day before testing. Twenty-four hours after habituation, mice were placed in the same box in the presence of 2 identical sample objects and were allowed to explore for 5 minutes. After an intersession interval (1 or 24 hours), mice were placed in the same box but 1 of the 2 objects was replaced by a novel object. Mice were allowed to explore for 5 minutes. Exploratory behavior was later assessed manually by an experimenter blinded to the treatment group. Exploration of an object was defined as the mouse sniffing the object or touching the object while looking at it (41). A minimal exploration time for both objects (total exploration time) during the test phase (10 seconds) was used. The amount of time taken to explore the novel object was expressed as percentage of the total exploration time and provides an index of recognition memory.

The Barnes maze test. The Barnes maze consisted of a circular open surface ( $90 \mathrm{~cm}$ in diameter) elevated to $90 \mathrm{~cm}$ by 4 wooden legs. There were 20 circular holes ( $5 \mathrm{~cm}$ in diameter) equally spaced around the perimeter, and positioned $2.5 \mathrm{~cm}$ from the edge of the maze. A wooden plastic escape box $(11 \times 6 \times 5 \mathrm{~cm})$ was positioned beneath 1 of the holes. Two neon lamps and a buzzer were used as aversive stimuli. The Any-Maze tracking system (Stoelting) was used to record the movement of mice on the maze. Extra-maze visual cues consisted of objects within the room and the experimenter. Mice were tested in groups of 10, and between trials they were placed into cages, which were placed in a dark room adjacent to the test room for the intertrial interval (20-30 minutes). All of the mice tested were trained with an escape hole located in the same location across trials and days (44). No habituation trial was performed. The acquisition phase consisted of 3 consecutive training days with 3 trials per day. On each trial a mouse was placed into a start tube located in the center of the maze, the start tube was raised, and the buzzer was turned on until the mouse entered the escape hole. After each trial, mice remained in the escape box for 60 seconds before being returned to their cage. Between trials the maze floor was cleaned with $10 \%$ ethanol in water to minimize olfactory cues. For each trial mice were given 3 minutes to locate the escape hole, after which they were guided to the escape hole or placed directly into the escape box if they failed to enter the escape hole. Four parameters of learning performance were recorded: (a) the latency to locate the escape hole (primary latency), (b) the latency to enter the escape hole (total latency), (c) the number of errors made, and (d) the distance traveled before locating the escape hole (44). Fifteen days after clodronate and around 30 days from the start of the first acquisition phase, mice were tested again for 3 consecutive days but the location of the escape hole was moved $90^{\circ}$ from its location during the first acquisition training phase.
Immunofluorescence. Mice were anesthetized with sodium pentobarbital $(120 \mathrm{mg} / \mathrm{kg}$, i.p.) and perfused transcardially with PBS followed by $4 \%$ paraformaldehyde (PFA) in PBS. Brain sections (thickness $40 \mu \mathrm{m}$ ) were cut through the whole brain using a microtome. Coronal sections were permeabilized in 0.5\% Triton-PBS and then blocked with $5 \%$ of normal donkey serum in $0.1 \%$ Triton-PBS. Sections were incubated with primary antibodies (CD206, clone MR5D3, rat polyclonal antibody: 1:200, Serotec; GLUT-1, rabbit polyclonal antibody: 1:200, Calbiochem; IBA1, rabbit polyclonal antibody: 1:500, Wako Chemicals; LYVE1, rabbit polyclonal antibody: 1:200, Abcam) at $4^{\circ} \mathrm{C}$ overnight. Sections were then incubated with a Cy5- or FITC-conjugated secondary antibody (1:200; Jackson ImmunoResearch Laboratories) and mounted on slides and examined with a Leica confocal microscope. $\mathrm{CD}_{20} 6^{+}$cells were counted by an investigator blinded to the treatment on 10 randomly selected fields ( $\times 20$ objective) within the somatosensory cortex and the hippocampus.

PVM number and vascular coverage. PVM coverage of cortical vessels was estimated by the ratio of PVM length/vessel length, identified by CD206 and GLUT-1, respectively. PVM number/vessel length was assessed as the number of PVMs per GLUT- $1^{+}$vessel length in each field according to vessel diameter in micrometers. For each animal, 10 randomly selected fields ( $\times 20$ objective), within the somatosensory cortex, were analyzed. All the analysis was done with Image (NIH).

Biotinylated ANGII labeling. ANGII- and saline-treated mice received a $50-\mu \mathrm{l}$ injection of biotinylated ANGII $(2 \mu \mathrm{g} / \mathrm{kg})$ into the left jugular vein. Five minutes after injection, mice were overdosed with sodium pentobarbital $(300 \mathrm{mg} / \mathrm{kg}$ ) and perfused through the left ventricle with (a) $20 \mathrm{ml}$ of heparin saline $(1,000 \mathrm{U} / \mathrm{ml})$, (b) $30 \mathrm{ml}$ of $3.75 \%$ acrolein (Polysciences) in 2\% PFA solution (SigmaAldrich), and (c) $50 \mathrm{ml}$ of $2 \%$ PFA solution. Coronal sections $(40 \mu \mathrm{m})$ from all mice were coprocessed for electron microscopic peroxidase using an avidin-biotin complex kit (Vector Laboratories). The peroxidase reaction product was visualized with $0.022 \% \mathrm{DAB}$ and $0.003 \% \mathrm{H}_{2} \mathrm{O}_{2}$. The tissue was then post-fixed with osmium tetroxide, dehydrated, and embedded in plastic. The cerebral cortex was isolated from the embedded tissue and cut to 70-nm sections with a Leica ultratome, collected onto 400 mesh copper grids, and counterstained with uranyl acetate and lead citrate. Cortical microvasculature was examined using a Technai transmission electron microscope outfitted with a CCD camera system (AMT Advantage). For immunofluorescence labeling, a solution containing biotinylated ANGII $(600 \mu \mathrm{l} ; 1 \mu \mathrm{M})(55)$ was infused into the right femoral vein, in both ANGII- and saline-treated mice, over 45 minutes. Mice were then overdosed with sodium pentobarbital $(300 \mathrm{mg} / \mathrm{kg})$ and perfused through the left ventricle with PBS followed by $4 \%$ PFA in PBS. Coronal sections $(40 \mu \mathrm{m})$ were cut using a vibratome and stained for CD206 and GLUT-1 as described above. Biotinylated ANGII was detected using a FITC-conjugated anti-biotin antibody (1:1,000; Jackson ImmunoResearch Laboratories). The specificity of the immunofluorescence was verified by omission of the FITC-conjugated antibody or by use of the anti-biotin antibody in the absence of biotinylated ANGII. Immunostaining was visualized by means of a Leica confocal microscope. CD20 $6^{+}$cells associated with biotinylated ANGII were counted by an investigator blinded to the treatment on randomly selected fields ( $\times 40$ objective) within the somatosensory cortex. 
Brain cell isolation for flow cytometry. Mice were transcardially perfused under deep anesthesia with $30 \mathrm{ml}$ of heparinized saline to remove blood cells, as described previously (65). Briefly, cerebral hemispheres were gently triturated in HEPES-HBSS buffer and then digested with $62.5 \mu \mathrm{g} / \mathrm{ml}$ Liberase DH (Roche Diagnostics) and 50 $\mathrm{U} / \mathrm{ml}$ DNase I (Worthington Biochemical). Cells were washed and subjected to discontinuous 70\%/25\% Percoll (GE Healthcare) density gradient centrifugation ( 25 minutes, $800 \mathrm{~g}, 4^{\circ} \mathrm{C}$ ). Enriched mononuclear cells were collected from the interphase and resuspended in FACS buffer for staining and flow cytometry analysis.

Flow cytometry. Rat monoclonal antibodies used for flow cytometry analysis include the following: CD45-APC (clone 30F-11), CD11bPE (clone M1/70), Ly6G-PerCP-Cy5.5 (clone 1A8), Ly6C-FITC (clone HK1.4), and CD2O6 (clone C068C2) (BioLegend). Erythrolysis was performed as described above, and afterward blood leukocytes were resuspended in buffer. Isolated brain cells and blood leukocytes were stained at $4^{\circ} \mathrm{C}$ with predetermined optimal concentrations of antibodies for 20 minutes and analyzed on an Accuri C6 flow cytometer (BD Biosciences) (65). For intracellular staining of CD206, cells were stained for surface markers, fixed and permeabilized using fixation and permeabilization buffers from eBioscience following the manufacturer's instructions. Cell populations were separated based on CD45 and Ly6C expression and further sorted by CD11b and CD206 or Ly6G expression. The cell markers used for cell type identification were as follows: brain macrophages, $\mathrm{CD} 45^{\mathrm{hi}} \mathrm{CD} 11 \mathrm{~b}^{+}$; brain PVMs, CD $45^{\text {hi }} \mathrm{CD} 11 \mathrm{~b}^{+} \mathrm{CD} 206^{+}$; and microglia, CD $45^{\text {int }} \mathrm{CD} 11 \mathrm{~b}^{+}$; blood lymphocytes, CD $45^{+} \mathrm{CD} 11 \mathrm{~b}^{-}$; blood monocytes, $\mathrm{CD} 45^{+} \mathrm{CD} 11 \mathrm{~b}^{+} \mathrm{Ly} 6 \mathrm{G}^{-}$; and blood polymorphonuclear leukocytes, $\mathrm{CD} 45^{+} \mathrm{CD} 11 \mathrm{~b}^{+} \mathrm{Ly}_{6 \mathrm{G}}{ }^{+}$. Appropriate isotype control antibodies, "fluorescence minus one" staining, and staining of negative populations were used to establish sorting parameters. Absolute cell numbers were recorded.

In vivo and in vitro cellular ROS measurement. ROS production was assessed in vivo by hydroethidine (HE) microfluorography (35). $\mathrm{HE}$ is a cell-permeant dye that is oxidized by superoxide (68). The fluorescence signal attributable to $\mathrm{HE}$ oxidation products reflects cumulative ROS production (35). Ringer solution containing HE ( $2 \mu \mathrm{mol} / \mathrm{l})$ was superfused over the somatosensory cortex. Mice were killed 60 minutes later, coronal brain sections were cut through cortex underlying the cranial window, and ROS-dependent fluorescence was quantified as described previously $(10,35)$.

$B B B$ permeability measurement. $\mathrm{BBB}$ permeability was assessed using sodium-fluorescein (NaF, MW $0.376 \mathrm{kDa} ; 100 \mu \mathrm{l}$ of $1 \%$ solution i.v.), fluorescein-dextran (FITC-dextran, MW $3 \mathrm{kDa} ; 100 \mu \mathrm{l}$ of $1 \%$ solution i.v.), or Evans blue (EB; MW $\sim 69 \mathrm{kDa}$ after binding to plasma albumin; $100 \mu$ of $2 \%$ solution i.v.) as previously described $(32,69)$. The markers were allowed to circulate for 20 minutes (NaF and FITC-dextran) or 1 hour (EB), and then mice were transcardially perfused with PBS. Brains were removed and brainstem and cerebellum were discarded. Tissue was homogenized in PBS, mixed with methanol (NaF and FITC-dextran) or trichloroacetic acid (EB), and centrifuged. The amount of NaF or FITC-dextran (485 nm excitation and $530 \mathrm{~nm}$ emission) in the supernatant was determined in duplicate using a fluorescence spectrophotometer. For EB measurements, samples were read on a plate reader (620 $\mathrm{nm}$ excitation, $680 \mathrm{~nm}$ emission), together with standards, and normalized by weight.

ANGII assay. Mice were killed, and blood was collected by cardiac puncture in a prechilled tube containing a mix of protease inhibitors. Plasma was collected and transferred to a prechilled tube and stored frozen at $-80^{\circ} \mathrm{C}$. Brains were collected and immediately frozen on dry ice. ANGII concentration was determined using a radioimmunoassay by the Hypertension Core Laboratory of the Wake Forest University School of Medicine (Winston-Salem, North Carolina, USA) (70).

Statistics. Sample size was determined according to power analysis. No animals were excluded, animals were randomly assigned to treatment and control groups, and analysis was performed in a blinded fashion. Intergroup differences were analyzed by a Student's $t$ test for single comparison or by 2-way ANOVA (Bonferroni's multiple comparison test) for multiple comparisons unless otherwise specified, as appropriate and indicated in the figure legends. Data are expressed as mean \pm SEM, and differences were considered statistically significant for $P$ less than 0.05 .

Study approval. All of the procedures were approved by the institutional animal care and use committee of Weill Cornell Medical College.

\section{Author contributions}

CI, JA, and GF designed the study; GF, YS, DL, LGB, HC, MMS, $\mathrm{GR}$, and MM conducted experiments and acquired data; GF and JA analyzed data; NVR provided reagents; and GF and CI wrote the manuscript.

\section{Acknowledgments}

This work was supported by grants R37-NS089323 and R01NS95441 from the NIH (to CI), a Scientist Development Grant from the American Heart Association (15SDG22760007) (to GF), and the Leducq Foundation (to CI, GF, and JA). The contribution from the Feil Family Foundation is gratefully acknowledged.

Address correspondence to: Costantino Iadecola, Feil Family Brain and Mind Research Institute, 407 East 61st Street, Room 303, New York, New York 10065, USA. Phone: 646.962.8279; E-mail: coi2001@med.cornell.edu.
1. Rahimi K, Emdin CA, MacMahon S. The epidemiology of blood pressure and its worldwide management. Circ Res. 2015;116(6):925-936.

2. Faraco G, Iadecola C. Hypertension: a harbinger of stroke and dementia. Hypertension. 2013;62(5):810-817.

3. Gorelick PB, et al. Vascular contributions to cognitive impairment and dementia: a statement for healthcare professionals from the American Heart Association/American Stroke Association. Stroke. 2011;42(9):2672-2713.

4. Iadecola C. The overlap between neurodegener- ative and vascular factors in the pathogenesis of dementia. Acta Neuropathol. 2010;120(3):287-296.

5. Lackland DT, et al. Factors influencing the decline in stroke mortality: a statement from the American Heart Association/American Stroke Association. Stroke. 2014;45(1):315-353.

6. Iadecola C. Neurovascular regulation in the normal brain and in Alzheimer's disease. Nat Rev Neurosci. 2004;5(5):347-360.

7. Attwell D, Buchan AM, Charpak S, Lauritzen M, Macvicar BA, Newman EA. Glial and neuronal control of brain blood flow. Nature.
2010;468(7321):232-243.

8. Zhao Z, Nelson AR, Betsholtz C, Zlokovic BV. Establishment and Dysfunction of the BloodBrain Barrier. Cell. 2015;163(5):1064-1078.

9. Andresen J, Shafi NI, Bryan RM. Endothelial influences on cerebrovascular tone. JAppl Physiol. 2006;100(1):318-327.

10. Capone C, et al. Central cardiovascular circuits contribute to the neurovascular dysfunction in angiotensin II hypertension. J Neurosci. 2012;32(14):4878-4886.

11. Kazama K, et al. Angiotensin II impairs neu- 
rovascular coupling in neocortex through NADPH oxidase-derived radicals. Circ Res. 2004;95(10):1019-1026.

12. Iadecola C. The pathobiology of vascular dementia. Neuron. 2013;80(4):844-866.

13. Romero JC, Reckelhoff JF. State-of-the-Art lecture. Role of angiotensin and oxidative stress in essential hypertension. Hypertension. 1999; 34(4 pt 2):943-949.

14. Kawada N, Imai E, Karber A, Welch WJ, Wilcox CS. A mouse model of angiotensin II slow pressor response: role of oxidative stress. J Am Soc Nephrol. 2002;13(12):2860-2868.

15. Johnson AW, Kinzenbaw DA, Modrick ML, Faraci FM. Small-molecule inhibitors of signal transducer and activator of transcription 3 protect against angiotensin II-induced vascular dysfunction and hypertension. Hypertension. 2013;61(2):437-442.

16. De Silva TM, Faraci FM. Effects of angiotensin II on the cerebral circulation: role of oxidative stress. Front Physiol. 2012;3:484.

17. Girouard H, Park L, Anrather J, Zhou P, Iadecola C. Cerebrovascular nitrosative stress mediates neurovascular and endothelial dysfunction induced by angiotensin II. Arterioscler Thromb Vasc Biol. 2007;27(2):303-309.

18. Goldmann T, et al. Origin, fate and dynamics of macrophages at central nervous system interfaces. Nat Immunol. 2016;17(7):797-805.

19. Moore KJ, Sheedy FJ, Fisher EA. Macrophages in atherosclerosis: a dynamic balance. Nat Rev Immunol. 2013;13(10):709-721.

20. Bechmann I, et al. Turnover of rat brain perivascular cells. Exp Neurol. 2001;168(2):242-249.

21. Vallières L, Sawchenko PE. Bone marrow-derived cells that populate the adult mouse brain preserve their hematopoietic identity. J Neurosci. 2003;23(12):5197-5207.

22. Brinker T, Stopa E, Morrison J, Klinge P. A new look at cerebrospinal fluid circulation. Fluids Barriers CNS. 2014;11:10.

23. Pizzolla A, et al. Reactive oxygen species produced by the NADPH oxidase 2 complex in monocytes protect mice from bacterial infections. JImmunol. 2012;188(10):5003-5011.

24. Yanagitani Y, et al. Angiotensin II type 1 receptor-mediated peroxide production in human macrophages. Hypertension. 1999;33(1 pt 2):335-339.

25. Zeisel A, et al. Brain structure. Cell types in the mouse cortex and hippocampus revealed by single-cell RNAseq. Science. 2015;347(6226):1138-1142.

26. Bechmann I, et al. Turnover of rat brain perivascular cells. Exp Neurol. 2001;168(2):242-249.

27. Polfliet MM, Goede PH, van Kesteren-Hendrikx EM, van Rooijen N, Dijkstra CD, van den Berg TK. A method for the selective depletion of perivascular and meningeal macrophages in the central nervous system. J Neuroimmunol. 2001;116(2):188-195.

28. Serrats J, Schiltz JC, García-Bueno B, van Rooijen N, Reyes TM, Sawchenko PE. Dual roles for perivascular macrophages in immune-to-brain signaling. Neuron. 2010;65(1):94-106.

29. Yu Y, Zhang ZH, Wei SG, Serrats J, Weiss RM, Felder RB. Brain perivascular macrophages and the sympathetic response to inflammation in rats after myocardial infarction. Hypertension.
2010;55(3):652-659.

30. Kazama K, Wang G, Frys K, Anrather J, Iadecola C. Angiotensin II attenuates functional hyperemia in the mouse somatosensory cortex. Am J Physiol Heart Circ Physiol. 2003;285(5):H1890-H1899.

31. Swirski FK, et al. Identification of splenic reservoir monocytes and their deployment to inflammatory sites. Science. 2009;325(5940):612-616.

32. Faraco G, et al. Hypertension enhances $A \beta$-induced neurovascular dysfunction, promotes $\beta$-secretase activity, and leads to amyloidogenic processing of APP. J Cereb Blood Flow Metab. 2016;36(1):241-252.

33. Dikalov SI, Nazarewicz RR. Angiotensin IIinduced production of mitochondrial reactive oxygen species: potential mechanisms and relevance for cardiovascular disease. Antioxid Redox Signal. 2013;19(10):1085-1094.

34. Girouard H, Park L, Anrather J, Zhou P, Iadecola C. Angiotensin II attenuates endotheliumdependent responses in the cerebral microcirculation through nox-2-derived radicals. Arterioscler Thromb Vasc Biol. 2006;26(4):826-832.

35. Capone C, Faraco G, Park L, Cao X, Davisson $\mathrm{RL}$, Iadecola $\mathrm{C}$. The cerebrovascular dysfunction induced by slow pressor doses of angiotensin II precedes the development of hypertension. Am J Physiol Heart Circ Physiol. 2011;300(1):H397-H407.

36. Schlager G, Sides J. Characterization of hypertensive and hypotensive inbred strains of mice. Lab Anim Sci. 1997;47(3):288-292.

37. Schlager G. Longevity in spontaneously hypertensive mice. Exp Gerontol. 1981;16(4):325-330.

38. Hartman RE, Kamper JE, Goyal R, Stewart JM, Longo LD. Motor and cognitive deficits in mice bred to have low or high blood pressure. Physiol Behav. 2012;105(4):1092-1097.

39. Duchemin S, Belanger E, Wu R, Ferland G, Girouard H. Chronic perfusion of angiotensin II causes cognitive dysfunctions and anxiety in mice. Physiol Behav. 2013;109:63-68.

40. Csiszar A, et al. Synergistic effects of hypertension and aging on cognitive function and hippocampal expression of genes involved in $\beta$-amyloid generation and Alzheimer's disease. Am J Physiol Heart Circ Physiol. 2013;305(8):H1120-H1130.

41. Leger $\mathrm{M}$, et al. Object recognition test in mice. Nat Protoc. 2013;8(12):2531-2537.

42. Barnes CA. Memory deficits associated with senescence: a neurophysiological and behavioral study in the rat. J Comp Physiol Psychol. 1979;93(1):74-104.

43. Harrison FE, Hosseini AH, McDonald MP. Endogenous anxiety and stress responses in water maze and Barnes maze spatial memory tasks. Behav Brain Res. 2009;198(1):247-251.

44. Harrison FE, Reiserer RS, Tomarken AJ, McDonald MP. Spatial and nonspatial escape strategies in the Barnes maze. Learn Mem. 2006;13(6):809-819.

45. Epelman S, Lavine KJ, Randolph GJ. Origin and functions of tissue macrophages. Immunity. 2014;41(1):21-35.

46. Abtin A, et al. Perivascular macrophages mediate neutrophil recruitment during bacterial skin infection. Nat Immunol. 2014;15(1):45-53.

47. Polfliet MM, et al. Meningeal and perivascular macrophages of the central nervous system play a protective role during bacterial meningitis. J Immunol. 2001;167(8):4644-4650.

48. Hawkes CA, McLaurin J. Selective targeting of perivascular macrophages for clearance of $\beta$-amyloid in cerebral amyloid angiopathy. Proc Natl Acad Sci U S A. 2009;106(4):1261-1266.

49. Thanopoulou K, Fragkouli A, Stylianopoulou F, Georgopoulos S. Scavenger receptor class B type I (SR-BI) regulates perivascular macrophages and modifies amyloid pathology in an Alzheimer mouse model. Proc Natl Acad Sci U S A. 2010;107(48):20816-20821.

50. Pires PW, Girgla SS, McClain JL, Kaminski NE, van Rooijen N, Dorrance AM. Improvement in middle cerebral artery structure and endothelial function in stroke-prone spontaneously hypertensive rats after macrophage depletion. Microcirculation. 2013;20(7):650-661.

51. Hillman EM. Coupling mechanism and significance of the BOLD signal: a status report. Annu Rev Neurosci. 2014;37:161-181.

52. Hill RA, Tong L, Yuan P, Murikinati S, Gupta S, Grutzendler J. Regional blood flow in the normal and ischemic brain is controlled by arteriolar smooth muscle cell contractility and not by capillary pericytes. Neuron. 2015;87(1):95-110.

53. Lund DD, Faraci FM, Miller FJ Jr. Heistad DD. Gene transfer of endothelial nitric oxide synthase improves relaxation of carotid arteries from diabetic rabbits. Circulation. 2000;101(9):1027-1033.

54. Capone C, Faraco G, Park L, Cao X, Davisson $\mathrm{RL}$, Iadecola $\mathrm{C}$. The cerebrovascular dysfunction induced by slow pressor doses of angiotensin II precedes the development of hypertension. Am J Physiol Heart Circ Physiol. 2011;300(1):H397-H407.

55. Biancardi VC, Son SJ, Ahmadi S, Filosa JA, Stern JE. Circulating angiotensin II gains access to the hypothalamus and brain stem during hypertension via breakdown of the blood-brain barrier. Hypertension. 2014;63(3):572-579.

56. Rose JM, Audus KL. Receptor-mediated angiotensin II transcytosis by brain microvessel endothelial cells. Peptides. 1998;19(6):1023-1030.

57. Mayhan WG, Faraci FM, Heistad DD. Disruption of the blood-brain barrier in cerebrum and brain stem during acute hypertension. Am JPhysiol. 1986;251(6 pt 2):H1171-H1175.

58. Davern PJ, Nguyen-Huu TP, La Greca L, Abdelkader A, Head GA. Role of the sympathetic nervous system in Schlager genetically hypertensive mice. Hypertension. 2009;54(4):852-859.

59. Palma-Rigo K, Jackson KL, Davern PJ, NguyenHuu TP, Elghozi JL, Head GA. Renin-angiotensin and sympathetic nervous system contribution to high blood pressure in Schlager mice. JHypertens. 2011;29(11):2156-2166.

60. McMaster WG, Kirabo A, Madhur MS, Harrison DG. Inflammation, immunity, and hypertensive endorgan damage. Circ Res. 2015;116(6):1022-1033.

61. Guzik TJ, et al. Role of the $\mathrm{T}$ cell in the genesis of angiotensin II induced hypertension and vascular dysfunction. JExp Med. 2007;204(10):2449-2460.

62. Te Riet L, van Esch JH, Roks AJ, van den Meiracker AH, Danser AH. Hypertension: renin-angiotensin-aldosterone system alterations. Circ Res. 2015;116(6):960-975.

63. Jennings JR, et al. Reduced cerebral blood flow 
response and compensation among patients with untreated hypertension. Neurology. 2005;64(8):1358-1365.

64. Levi Marpillat N, Macquin-Mavier I, Tropeano AI, Bachoud-Levi AC, Maison P. Antihypertensive classes, cognitive decline and incidence of dementia: a network meta-analysis. J Hypertens. 2013;31(6):1073-1082.

65. Garcia-Bonilla L, Racchumi G, Murphy M, Anrather J, Iadecola C. Endothelial CD36 contributes to postischemic brain injury by promot- ing neutrophil activation via CSF3. J Neurosci. 2015;35(44):14783-14793.

66. Livak KJ, Schmittgen TD. Analysis of relative gene expression data using real-time quantitative PCR and the $2(-\Delta \Delta C(T))$ method. Methods. 2001;25(4):402-408.

67. Faraco G, Moraga A, Moore J, Anrather J, Pickel VM, Iadecola C. Circulating endothelin- 1 alters critical mechanisms regulating cerebral microcirculation. Hypertension. 2013;62(4):759-766.

68. Robinson KM, et al. Selective fluorescent imaging of superoxide in vivo using ethidium-based probes. Proc Natl Acad Sci U S A. 2006;103(41):15038-15043.

69. Carman AJ, Mills JH, Krenz A, Kim DG, Bynoe MS. Adenosine receptor signaling modulates permeability of the blood-brain barrier. J Neurosci. 2011;31(37):13272-13280.

70. Nakamoto H, Ferrario CM, Fuller SB, Robaczewski DL, Winicov E, Dean RH. Angiotensin-(1-7) and nitric oxide interaction in renovascular hypertension. Hypertension. 1995;25(4 pt 2):796-802. 